\title{
ROTATIONAL AND VIBRATIONAL DYNAMICS OF INTERSTITIAL MOLECULAR HYDROGEN
}

\author{
T. Yildirim \\ National Institute of Standards and Technology, Gaithersburg, Md 20899 \\ A. B. Harris \\ Department of Physics and Astronomy, University of Pennsylvania, Philadelphia, PA 19104 \\ and National Institute of Standards and Technology, Gaithersburg, Md 20899
}

(November 8, 2018)

\begin{abstract}
The calculation of the hindered roton-phonon energy levels of a hydrogen molecule in a confining potential with different symmetries is systematized for the case when the rotational angular momentum $J$ is a good quantum number. One goal of this program is to interpret the energy-resolved neutron time of flight spectrum previously obtained for $\mathrm{H}_{2} \mathrm{C}_{60}$. This spectrum gives direct information on the energy level spectrum of $\mathrm{H}_{2}$ molecules confined to the octahedral interstitial sites of solid $\mathrm{C}_{60}$. We treat this problem of coupled translational and orientational degrees of freedom a) by construction of an effective Hamiltonian to describe the splitting of the manifold of states characterized by a given value of $J$ and having a fixed total number of phonon excitations, b) by numerical solutions of the coupled translation-rotation problem on a discrete mesh of points in position space, and c) by a group theoretical symmetry analysis. Results obtained from these three different approaches are mutually consistent. The results of our calculations explain several hitherto uninterpreted aspects of the experimental observations, but show that a truly satisfactory orientational potential for the interaction of an $\mathrm{H}_{2}$ molecule with a surrounding array of $\mathrm{C}$
\end{abstract}


atoms has not yet been developed.

Typeset using REVTEX 


\section{INTRODUCTION}

The study of rotational and vibrational dynamics of guest molecules (i.e. $\mathrm{CO}, \mathrm{O}_{2}, \mathrm{H}_{2}$, etc) trapped in porous media such as fullerenes, zeolites, and graphite has recently become an

active subject both experimentally and theoretically. ${ }^{1-5}$ This is because such studies can yield valuable information about the host-guest interactions which could be important for several technical applications such as gas separation and hydrogen storage. ${ }^{1-3}$ In particular hydrogen molecules trapped in interstitial cavities in solid $\mathrm{C}_{60}$ as well as hydrogen molecules embedded in nanotube ropes are of interest due to quantum behavior of hydrogen molecules in quasi zero and one dimensional sites. ${ }^{2,3,5}$

In this paper, we develop a detailed analysis of coupled rotational and vibrational dynamics of a molecular hydrogen encapsulated in a solid using numerical, perturbative, and group theoretical methods. In particular we will be interested in what one might call the "weak coupling limit," when the interaction between molecular rotations and center-of-mass translations is weak enough that the rotational angular momentum quantum number $J$ is a good quantum number. This limit is almost never satisfied except for very light molecules like hydrogen or deuterium. The energy levels of a free rotator are

$$
E_{J}=B J(J+1)
$$

where $B=\hbar^{2} /(2 I)$, where $I$ is the moment of inertia of the molecule and $E_{J}$ is $(2 J+1)$-fold degenerate. For $\mathrm{H}_{2}$ the rotational constant $2 B$ has the value $60 \mathrm{~cm}^{-1}, 14.7 \mathrm{meV}$, or $B / k=85$ $\mathrm{K}$ (and the corresponding values for $\mathrm{D}_{2}$ are half as large), so that the energy separation between different $J$ levels is large enough that often $J$ is a good quantum number. This is certainly true for solids consisting of these molecules unless the pressure is quite large. (For a review of the properties of the hydrogen molecule and solid hydrogen see Ref. 6.)

We have been led to consider this phenomenon in view of an experimental study of energy spectra of $\mathrm{H}_{2}$ and $\mathrm{D}_{2}$ inserted into the octahedral interstitial sites in solid $\mathrm{C}_{60}$ carried out by neutron time of flight techniques. ${ }^{5}$ In considering this phenomenon we should keep in mind the following experimental facts concerning the host solid of $\mathrm{C}_{60}$. The centers of the $\mathrm{C}_{60}$ molecules form an fcc lattice. ${ }^{7}$ At temperature above $T_{c}$, where $T_{c}$ is about $260 \mathrm{~K}$, the 
molecules are orientationally disordered. At $T=T_{c}$ long range orientational ordering occurs ${ }^{8}$ and the molecules are ordered into four sublattices as described by $\mathrm{Pa} \overline{3}$ symmetry. ${ }^{9-12}$ In the orientationally disordered phase the local symmetry at the the octahedral interstitial site is indeed that of the point group $\mathrm{O}_{h}$. In the presence of orientational ordering the symmetry of what was the "octahedral" interstitial site is now reduced to a uniaxial symmetry, specifically that of point group $\mathrm{S}_{6} \cdot{ }^{13}$ In experiments, hydrogen molecules are stable in the octahedral interstitial site only for temperatures well below $T_{c}$ (where the interstitial site does not actually have octahedral symmetry).

While a general understanding of the time-of-flight experiments was presented, ${ }^{5}$ some of the finer details of the experiment remained unexplained. For instance, the shift in the energy associated with ortho-para $(J=1 \rightarrow J=0)$ conversion in the interstitial relative to its value for free molecules was not understood. Also the feature in the energy gain spectrum at about twice the ortho-para conversion energy was not unambiguously identified. These issues are both addressed in this paper. More generally we give a calculation of the cross section for neutron energy loss for comparison with the observed time-of-flight spectrum. For that purpose we need not only to consider the cross section for para-ortho conversion as compared to phonon creation, but also to calculate the phonon excitations of $(J=1)$ molecules. These calculations require us to develop and implement a scheme for treating coupled translations and rotations. In this paper we present a systematic analysis of the simplest case of this coupling which occurs when the quantum number $J$ characterizing free rotation remains a good quantum number. In that case the well-known numerical schemes for solving the translation problem can be easily extended to include the effect of the coupling to rotations. In addition, we also give analytic expressions obtained by treating this coupling within perturbation theory. As we will see, this analytic development enables us to interpret many of the numerical results in a meaningful way. In addition, we analyze in detail various simplified models which illustrate our group theoretical analysis of the symmetry present in the system of coupled translations and rotations. This analysis indicates that arguments for the degeneracies of coupled translationrotation modes based on simple classical concepts are incorrect. In summary: in this paper we present an analysis based on numerical, perturbative, and group theoretical methods. 
Up to now there have not been many theoretical studies of energy levels in such irregular geometries like the octahedral interstitial sites in $\mathrm{C}_{60}$. A notable exception is the work of van der Avoird and collaborators ${ }^{14}$ on $\mathrm{CO}$ in $\mathrm{C}_{60}$. That work examined an even more complicated situation in which the rotational and translation degrees of freedom interacted strongly. As a result, the problem was analyzed numerically. In contrast, for the present problem FitzGerald et al. ${ }^{5}$ applied a number of analytic and semi-analytic techniques to the theoretical study the spectra of hydrogen molecules in $\mathrm{C}_{60}$. This paper may be regarded as an extension and systematization of their approach.

\section{GENERAL FORMULATION}

Clearly the first step is to establish a satisfactory potential for the intercalated hydrogen molecule. This potential function $V(\mathbf{r}, \Omega)$ gives the energy of a hydrogen molecule whose center of mass is at $\mathbf{r}$ and whose orientation is specified by $\Omega \equiv(\theta, \phi)$. A convenient starting point is to use an atom-atom potential ${ }^{15}$ to describe the interaction between each of the two hydrogen atoms and the atoms in the confining structure. Unless otherwise indicated, all the results reported in this paper are obtained from the same WS77 potential, ${ }^{15}-A / r^{6}+B \exp (-C r)$, that is used in Ref. 5 (where $A=5.94 \mathrm{eV} \AA^{6}, B=678.2 \mathrm{eV}$, and $C=3.67 \AA^{-1}$ ).

In this paper we will mainly consider the octahedral interstitial site in solid $\mathrm{C}_{60}$, but many of the considerations apply with slight modification to molecules confined within other structures such as single wall carbon nanotubes. ${ }^{16}$ The determination of the potential $V(\mathbf{r}, \Omega)$ for $\mathrm{H}_{2}$ in solid $\mathrm{C}_{60}$ is discussed in Appendix A. From the numerical evaluation of this potential we have extracted the expansion coefficients when it is written in the following canonical form:

$$
V(\mathbf{r}, \Omega)=V_{0}(\mathbf{r})+\sum_{l=2,4, \ldots} \sum_{m=-l}^{l} A_{l}^{m}(\mathbf{r}) Y_{l}^{m}(\Omega) .
$$

We assume (and it is generally true) that the orientational energies which are relevant are much less than the smallest energy difference between successive $J$ levels of a molecule (10B for an ortho molecule and $6 B$ for a para molecule). Accordingly, we may consider only that part of the potential which is diagonal in $J$. When the potential is written in the form of Eq. (2), it 
is easy to implement the truncation to terms diagonal in $J$. So for a fixed value of $J$ we have the Hamiltonian $\mathcal{H}_{J}$ as

$$
\begin{aligned}
\mathcal{H}_{J} & =\frac{p^{2}}{2 m}+V_{0}(\mathbf{r})+B J(J+1) \mathcal{I}+\sum_{l=2,4, \ldots} \sum_{\mu, \mu^{\prime}} A_{l}^{\mu-\mu^{\prime}}(\mathbf{r})\left[|J \mu\rangle\left\langle J \mu\left|Y_{l}^{\mu-\mu^{\prime}}(\Omega)\right| J \mu^{\prime}\right\rangle\left\langle J \mu^{\prime}\right|\right] \\
& =-\frac{\hbar^{2}}{2 m} \nabla^{2}+V_{0}(\mathbf{r})+B J(J+1)+V_{J}(\mathbf{r}),
\end{aligned}
$$

where $V_{J}(\mathbf{r})$ is the orientationally dependent part of the potential (the terms involving $A_{l}^{m}$ ) and $\mathcal{I}$ is the unit operator. Furthermore we consider the angular-dependent term in this expansion to be a perturbation on the first term, $V_{0}(\mathbf{r})$. For each value of $J$ the Hamiltonian $\mathcal{H}_{J}$ will give a manifold of states which is the direct product of a manifold corresponding to various numbers of localized phonons being excited with the manifold of $(2 J+1)$ states having different values of $m_{J}$. An important simplification is that spherical harmonics with $l>2 J$ have no nonzero matrix elements in the manifold of states of angular momentum $J$.

Note that apart from the kinetic energy, this Hamiltonian is a strictly local operator. Thus we solve the eigenvalue problem on a discrete mesh of points on a cube centered at the octahedral site when the wave function is required to vanish on the boundary of the cube. Each edge of the cube is taken to be $[-L, L]$ with mesh point spacing of $d L$. In this scheme the wave function at each mesh point is a $(2 J+1)$-component vector. We are mainly concerned with the manifold $J=0$ and $J=1$, in which case the problem is numerically not significantly harder than for a scalar problem. Even though the resulting matrix size is very large, it is a block band matrix and is very sparse. The numerical results reported here were obtained from $\mathrm{L}=1.65 \AA$ and $d L=0.075 \AA$, which requires diagonalization of a matrix $n \times n$ where $n=273375)$. However we confirmed that a coarse mesh points with $L=1.2 \AA$ and $d L=0.17 \AA$, gives almost the same results (where the matrix size is $n=14,739$ ). The large sparse matrix eigenvalue problem is solved using the package ARPACK. ${ }^{17}$

Since numerical results sometimes do not provide complete insight into the nature of the solutions, we have also used perturbation theory to understand the results. In this approach we treat $V_{J}(\mathbf{r})$ in Eq. (3) as the perturbation. The unperturbed problem, apart from the additive energy $B J(J+1)$ is thus that for translations of the spherical $(J=0)$ molecule. This spectrum is not too different from that of a three-dimensional harmonic oscillator. Accordingly, 
to qualitatively interpret our more accurate numerical results, we apply perturbation theory in which we develop an effective Hamiltonian ${ }^{18}$ to describe the splitting of this manifold which is characterized by a value of $J$ and of $N$, the total number of phonon excitations. (The perturbative effects due to coupling between manifolds of different $J$ is negligibly small for hydrogen in $\mathrm{C}_{60} \cdot{ }^{5}$ ) This effective Hamiltonian is a matrix of dimensionality $D$, where $D=$ $(2 J+1)(N+1)(N+2) / 2$ and schematically is of the form

$$
\mathcal{H}(N, J)=B J(J+1) \mathcal{I}+\mathcal{H}_{\text {phonon }}+V_{J}^{\mathrm{DIAG}}-V_{J}^{\mathrm{OFF}} \frac{1}{\mathcal{E}} V_{J}^{\mathrm{OFF}}
$$

where $\mathcal{H}_{\text {phonon }}$ gives the energy of the various states with a total of $N$ phonons. These energies are just those calculated for a $(J=0)$ molecule. Also $V_{J}^{\text {DIAG }}$ is the part of $V_{J}$ which is diagonal with respect to the number of phonons, $V_{J}^{\mathrm{OFF}}$ is the part of $V_{J}$ which is off-diagonal with respect to the number of phonons, and $\mathcal{E}$ is the change in phonon energy caused by $V_{J}^{\mathrm{OFF}}$.

This effective Hamiltonian is defined by its matrix elements as

$$
\begin{aligned}
\langle N, \alpha ; & \left.J, M|\mathcal{H}(J, N)| N, \alpha^{\prime} ; J, M^{\prime}\right\rangle=\left[B J(J+1)+E_{N, \alpha}\right] \delta_{\alpha, \alpha^{\prime}} \delta_{M, M^{\prime}} \\
& +\sum_{l=1}^{J}\left\langle N, \alpha\left|A_{2 l}^{M-M^{\prime}}(\mathbf{r})\right| N, \alpha^{\prime}\right\rangle\left\langle J M\left|Y_{2 l}^{M-M^{\prime}}(\Omega)\right| J M^{\prime}\right\rangle \\
+ & \sum_{N^{\prime} \neq N} \sum_{l, l^{\prime}=1}^{J} \sum_{\mu} \sum_{\beta=1}^{k_{N^{\prime}}}\left[E_{N, \alpha}-E_{N^{\prime}, \beta}\right]^{-1}\left\langle N, \alpha\left|A_{2 l}^{M-\mu}(\mathbf{r})\right| N^{\prime}, \beta\right\rangle\left\langle N^{\prime}, \beta\left|A_{2 l^{\prime}}^{\mu-M^{\prime}}(\mathbf{r})\right| N, \alpha^{\prime}\right\rangle \\
& \left\langle J M\left|Y_{2 l}^{M-\mu}(\Omega)\right| J \mu\right\rangle\left\langle J \mu\left|Y_{2 l^{\prime}}^{\mu-M^{\prime}}(\Omega)\right| J M^{\prime}\right\rangle,
\end{aligned}
$$

where $k_{N}=(N+1)(N+2) / 2$ and the states with $N$ phonons are labeled $N$, $\alpha$, where $\alpha$ runs from 1 to $k_{N}$.

We now briefly discuss how the $A_{l}^{m}$ 's of Eq. (2) are obtained from the atom-atom potential between each $\mathrm{H}$ atom and each carbon atom. Here we will assume that the atom-atom potential is of the form $F\left(\left|\mathbf{r}_{i}-\mathbf{r}_{H}\right|\right)$, where $\mathbf{r}_{i}$ and $\mathbf{r}_{H}$ are the displacements of the $i$ th carbon and of the $\mathrm{H}$ atom, respectively, relative to the center of the $\mathrm{H}_{2}$ molecule. For this form of potential, we show in Appendix A that

$$
A_{2}^{m}=2 \pi \sum_{i} Y_{2}^{m}\left(\hat{\mathbf{r}}_{i}\right)^{*} \int_{-1}^{1}\left(3 x^{2}-1\right) F\left(\left[r_{i}^{2}-\frac{1}{4} \rho^{2}+\rho x r_{i}\right]^{1 / 2}\right) d x,
$$

where $\rho$ is the separation between $\mathrm{H}$ atoms in the $\mathrm{H}_{2}$ molecule and the sum over $i$ is over all relevant neighboring carbon atoms. It is instructive to expand this expression in $\rho / r_{i}$, which yields 


$$
A_{2}^{m}=\frac{1}{4} \rho^{2}\left(\frac{8 \pi}{15}\right) \sum_{i}\left(F^{\prime \prime}-\frac{F^{\prime}}{r_{i}}\right) Y_{2}^{m}\left(\hat{\mathbf{r}}_{i}\right)+o\left(\rho^{4} / r_{i}^{4}\right)
$$

where $F^{\prime \prime}$ and $F^{\prime}$ are the second and first derivatives of $F\left(r_{i}\right)$.

This expansion is good enough to reproduce most of the results discussed in this paper. We note that the $A_{l}^{m}$ 's (i.e. the orientational potential) are zero for a harmonic potential (i.e.

$\left.F\left(r_{i}\right)=\frac{1}{2} k r_{i}^{2}\right)$ because the prefactor $\left(F^{\prime \prime}-F^{\prime} / r_{i}\right)$ is zero. This can be also seen easily as follows. Assuming an atom-atom potential between each $\mathrm{H}$ atom and the $\mathrm{C}$ atoms in the adjacent $\mathrm{C}_{60}$ molecules, we may write the potential of an $\mathrm{H}_{2}$ molecule as

$$
V(\mathbf{r} ; \Omega)=V_{a}\left(\mathbf{r}+\frac{1}{2} \rho \hat{n}\right)+V_{a}\left(\mathbf{r}-\frac{1}{2} \rho \hat{n}\right)
$$

where $V_{a}$ is the potential of a single atom due to the entire octahedral cage in which it is confined, $\hat{n}$ is a unit vector along the axis of the molecule, and $\rho$ is the separation between atoms in the molecule. As we shall see, the total potential is nearly isotropic. So we write

$$
V_{a}(\mathbf{r})=\frac{1}{2} k r^{2}+\delta r^{4}
$$

When we substitute this into Eq. (8), we obtain the result

$$
V(\mathbf{r} ; \Omega)=V_{0}(r)+2 \delta\left[r^{2} \rho^{2} \cos ^{2} \theta_{r, n}-\frac{1}{3}\right]
$$

where $\theta_{r, n}$ is the angle between the vectors $\mathbf{r}$ and $\hat{n}$ and $V_{0}$ is independent of $\theta_{r, n}$. The point is that the orientationally dependent part of the interaction depends on the anharmonicity: for a purely harmonic and isotropic interaction $V_{a}$, the total potential energy is independent of the molecular orientation. Thus we expect rotation-translation coupling to be weak. On the other hand in nanotubes, where the quadratic term is anisotropic, this coupling will be more important. ${ }^{16}$

\section{ENERGY SPECTRUM OF A $(J=0) \mathbf{H}_{2}$ MOLECULE}

We start by considering the eigenvalue spectrum of $\mathcal{H}_{0}$ in which the orientational dependence of the potential is neglected. In this approximation, apart from the additive constant $B J(J+1)$, the total energy (rotational plus translational) is the same as that of a $(J=0)$ molecule. For 
most purposes a $(J=0)$ molecule may be considered to be a spherical molecule because the orientational wave function $Y_{0}^{0}(\Omega)$ is uniform over all orientations. Each eigenfunction of $\mathcal{H}_{0}(J)$ is the product of a rotational function taken from the manifold of $2 J+1$ degenerate orientational wave functions and a translational wave function which represents an eigenfunction for a spherical molecule confined to a cage. These translational wave functions satisfy

$$
\mathcal{H}_{0} \psi_{k}(\mathbf{r})=\left[\frac{p^{2}}{2 m}+V_{0}(\mathbf{r})\right] \psi_{k}(\mathbf{r})=E_{k} \psi_{k}(\mathbf{r})
$$

where $V_{0}(\mathbf{r})$ is the potential discussed in Appendix A. The index $k$ labels states which we might otherwise label by three indices, each quantum number characterizing the number of excitations in each direction. Note that these unperturbed solutions do not involve the coupling between rotations and translations. As discussed above, these eigenfunctions were obtained by converting the continuum equation (11) into a into a discrete equation on a mesh of points and solving the resulting matrix eigenvalue problem using a sparse matrix routine. ${ }^{17}$

Since it happens that the energy levels and eigenfunctions we obtained numerically are not qualitatively different from those of a spherical harmonic oscillator, we first study the energy spectrum as perturbations, $\delta, \kappa$, and $\lambda$, are sequentially turned on in the following potential:

$$
V(r)=\frac{1}{2} k r^{2}+\delta r^{4}+\kappa\left(x^{4}+y^{4}+z^{4}-\frac{3}{5} r^{4}\right)+\lambda(x y+y z+z x) .
$$

Fig. 1 shows the evolution of the energy spectrum as perturbations are sequentially introduced which take the spherical harmonic oscillator into the actual lower symmetry of a molecule in an octahedral interstitial site. In the left-most panel we show the energy levels for a spherical harmonic oscillator, with $\hbar \omega$ adjusted to correspond to the single-phonon levels of $\mathrm{H}_{2}$ in an octahedral interstitial site in $\mathrm{C}_{60}$. Note that the levels are highly degenerate because the energy depends only on the total number of quanta. The symmetry of the Hamiltonian is $U(3)$, the group of unitary three dimensional matrices. We now add to this potential an anharmonic term of the form $\delta r^{4}$. This perturbation lowers the symmetry to that of the rotation group in three dimensions. As is well known, each eigenfunction in a generic spherically symmetric potential can be labeled by the magnitude of the orbital angular momentum, $L$. Thus the single phonon levels are unsplit by this anharmonic perturbation and are now labeled as angular momentum $L=1$ states, whereas the two phonon levels split into a manifold of five $L=2$ states and one 
$L=0$ state and similarly for states with more than two phonons. In Fig. 1 we have taken the constant $\delta$ to be that which best describes the anharmonicity of $\mathrm{H}_{2}$ in $\mathrm{C}_{60}$. The energies of the perturbed levels are given in Table I.

Next, we consider what happens when the spherical oscillator potential is augmented by a cubic symmetry potential of the form $\kappa\left(x^{4}+y^{4}+z^{4}-\frac{3}{5} r^{4}\right)$. This potential is appropriate for a spherical molecule in an octahedral interstitial when the $\mathrm{C}_{60}$ are orientationally disordered and have an Fm3m crystal structure. ${ }^{7}$ The degeneracy associated with spherical symmetry is lifted, ${ }^{19}$ but as shown here one retains cubic symmetry, so the three one-phonon states which transform as $x, y$, and $z$ are degenerate. The two-phonon states are of three different symmetries. One $\left(t_{2 g}\right)$ transforms like $x y, x z$, and $y z$. This is the lowest level. The next highest level is the s-wave symmetric combination which transforms like $x^{2}+y^{2}+z^{2}$. Then one has a doublet of d-wave $\left(e_{2 g}\right)$ symmetry. This classification scheme is continued in the higher-energy levels. Although we are not dealing with harmonic phonons, it is still useful to consider manifolds characterized by the quantum numbers $J$ and $N$, which are respectively the rotational angular momentum and the total number of phonons, at least up to $N=3$. Quantitative results are given in Table II.

Finally, in the rightmost panel of Fig. 1 we show the further reduction in degeneracy which occurs when the octahedral interstitial is surrounded by $\mathrm{C}_{60}$ molecules which have the long range order associated with the $\mathrm{Pa} \overline{3}$ crystal structure. ${ }^{9-12}$ In this case, each interstitial is uniaxial (with symmetry $\mathrm{S}_{6}$ ) rather than octahedral. Accordingly, we introduce a potential of the form $\lambda(x y+y z+z x) \equiv \frac{1}{2} \lambda\left(3 \xi^{2}-r^{2}\right)$, where the $\xi$ axis is taken to lie along the three-fold axis of the interstitial site. There are four symmetry related interstitial sites, each of which has its three-fold axis along a different $[1,1,1]$ direction. The resolution of degeneracy in the presence of this uniaxial perturbation is also given in Table II. In all these cases, no interactions between rotations and translations are involved.

We have solved the eigenvalue problem of Eq. (11) on a mesh of points and obtained the results given in Table III. Results labeled "Octahedral" are those for the orientationally disordered phase, where each $\mathrm{C}_{60}$ molecule is replaced by a sphere of carbon atoms as is discussed in Ref. 5. Since these numerical results lead to manifolds of energy levels associated with a given 
number of phonons and the degeneracies of these manifolds are as expected from our general discussion above, we conclude that the potential seen by a spherical $\mathrm{H}_{2}$ molecule in the lowlying phonon levels is not very different from that of a spherical harmonic oscillator. However as noted in Ref. 5, the effective harmonic potential must be taken to be a self-consistently renormalized potential to take account of the larger zero-point motion.

\section{ENERGY SPECTRUM OF A $J=1$ MOLECULE}

We now discuss the energy spectrum of an ortho molecule with $(J=1)$. As we have seen for $(J=0)$ molecules, our numerical results indicate that for $N$ up to, say, three, one can clearly identify the manifold of $N$ phonons. We therefore discuss the systematics of these manifolds.

\section{A. Zero-Phonon Manifold}

We first consider the case of $J=1$ with $N=0$ phonons. This manifold is described by the effective Hamiltonian

$$
\mathcal{H}=[2 B+\Delta E] \mathcal{I}+\delta\left[J_{z}^{2}-\frac{2}{3}\right]
$$

The splitting $\delta$ must be zero when $\mathrm{C}_{60}$ is orientationally disordered. From Eq. (5) one sees that because the spherical harmonics are traceless, the average energy shift, $\Delta E$, has nonzero contributions only from terms which involve coupling to excited phonon states. (In Ref. 5 a negligibly small shift was found due to effects off-diagonal in $J$ which we ignore here.) From Eq. (5) we find that

$$
\Delta E=-\frac{1}{3} \sum_{N \neq 0, \alpha} E_{N, \alpha}^{-1} \sum_{\mu, \tau}\left|\left\langle 0,1\left|A_{2}^{\tau}(\mathbf{r})\right| N, \alpha\right\rangle\right|^{2}\left|\left\langle 1(\mu+\tau)\left|Y_{2}^{\tau}(\Omega)\right| 1 \mu\right\rangle\right|^{2}
$$

To implement this equation, we first construct $A_{l}^{m}(\mathbf{r})$ as discussed in Eq. (6). Then matrix elements of $A_{2}^{M}(\mathbf{r})$ are taken between phonon states for a $J=0$ molecule which we obtained previously and which are labeled $N, \alpha(|0,1\rangle$ being the phonon ground state). Thereby we obtained the results given in Tables IV and V. In Eq. (14) the matrix elements of spherical 
harmonics $Y_{2}^{M}(\Omega)$ are taken between orientational states labeled by $J$ and $J_{z}$. To evaluate $\Delta E$ we use

$$
\sum_{\mu}\left|\left\langle 1(\mu+\tau)\left|Y_{2}^{\tau}(\Omega)\right| 1 \mu\right\rangle\right|^{2}=\frac{3}{10 \pi}
$$

so that

$$
\Delta E=-\frac{1}{10 \pi} \sum_{N \neq 0, \alpha} E_{N, \alpha}^{-1} \sum_{\tau}\left|\left\langle 0,1\left|A_{2}^{\tau}(\mathbf{r})\right| N, \alpha\right\rangle\right|^{2} .
$$

In appendix $\mathrm{B}$ we give a model calculation of an $\mathrm{H}_{2}$ molecule in a spherical cavity from which we evaluate Eq. (16) to give $\Delta E=-0.14 \mathrm{meV}$. In this calculation the translational wave functions are assumed to be those of a harmonic oscillator with $\left\langle r^{2}\right\rangle=0.1875 \AA^{2}$. As noted, the result is very sensitive to the value used for $\left\langle r^{2}\right\rangle$. For octahedral symmetry (i. e. for orientationally disordered $\mathrm{C}_{60}$ ) we evaluate Eq. (16) using the data in Table IV. Thereby we find a shift $\Delta E=-0.133 \mathrm{meV}$. The same approach using our numerical solutions for the phonon states of a $(J=0)$ molecule for the orientationally ordered $\mathrm{Pa} \overline{3}$ phase yields the result, $\Delta E=-0.141 \mathrm{meV}$, compared to the experimental value ${ }^{5} \Delta E=-0.35 \mathrm{meV}$. Again we mention that a small change in parameters could easily lead to a much larger calculated value of $\Delta E$. From the numerical solution for the three component wave function of a $(J=1)$ molecule on a mesh of points, we obtained the value $\Delta E=-0.16$ for the $\mathrm{Pa} \overline{3}$ phase. The various numerical results for $\Delta E$ are summarized in Table VI.

From Eq. (5) we also find the splitting (in the $\mathrm{Pa} \overline{3}$ phase) to be

$$
\begin{aligned}
\delta= & -\frac{3\left\langle 0,1\left|A_{2}^{0}\right| 0,1\right\rangle}{\sqrt{20 \pi}} \\
& +\frac{3}{20 \pi} \sum_{e} E(e)^{-1}\left[\left|\left\langle 0,1\left|A_{2}^{0}(\mathbf{r})\right| e\right\rangle\right|^{2}+\left|\left\langle 0,1\left|A_{2}^{1}(\mathbf{r})\right| e\right\rangle\right|^{2}-2\left|\left\langle 0,1\left|A_{2}^{2}(\mathbf{r})\right| e\right\rangle\right|^{2}\right],
\end{aligned}
$$

where the quantization axis is taken to lie along the threefold axis of symmetry of the interstitial site. Using the matrix elements given in Table IV, we find that the contribution to the splitting $\delta$ comes almost exclusively from the diagonal term $\left\langle 0,1\left|A_{2}^{0}\right| 0,1\right\rangle$ and we obtain the results listed in Table VI. 


\section{B. One-Phonon Manifold}

\section{Numerical Results}

Next we consider the manifold $J=1$ with $N=1$ phonon. Again only $Y_{l}^{m}$ with $l=2$ contributes, so that we may write

$$
\begin{aligned}
\mathcal{H}(1,1)_{\alpha \mu ; \alpha^{\prime} \mu^{\prime}} \equiv & \left\langle\alpha \mu|\mathcal{H}(N=1, J=1)| \alpha^{\prime} \mu^{\prime}\right\rangle \\
= & {\left[2 B+E_{0}+\hbar \omega_{\alpha}\right] \delta_{\mu, \mu^{\prime}} \delta_{\alpha, \alpha^{\prime}}+\left\langle 1 \alpha\left|A_{2}^{\mu-\mu^{\prime}}(\mathbf{r})\right| 1 \alpha^{\prime}\right\rangle\left\langle 1 \mu\left|Y_{2}^{\mu-\mu^{\prime}}(\Omega)\right| 1 \mu^{\prime}\right\rangle } \\
& -\sum_{N^{\prime} \neq 1} \sum_{\alpha^{\prime \prime}=1}^{k_{N^{\prime}}} \sum_{\mu^{\prime \prime}} \frac{1}{E_{N^{\prime}, \alpha^{\prime \prime}}-E_{1, \alpha}}\left\langle 1 \alpha\left|A_{2}^{\mu-\mu^{\prime \prime}}(\mathbf{r})\right| N^{\prime} \alpha^{\prime \prime}\right\rangle\left\langle N^{\prime} \alpha^{\prime \prime}\left|A_{2}^{\mu^{\prime \prime}-\mu^{\prime}}(\mathbf{r})\right| 1 \alpha^{\prime}\right\rangle \\
& \quad \times\left\langle 1 \mu\left|Y_{2}^{\mu-\mu^{\prime \prime}}(\Omega)\right| 1 \mu^{\prime \prime}\right\rangle\left\langle 1 \mu^{\prime \prime}\left|Y_{2}^{\mu^{\prime \prime}-\mu^{\prime}}(\Omega)\right| 1 \mu^{\prime}\right\rangle .
\end{aligned}
$$

This is a $9 \times 9$ matrix, which gives the $9(J=1, N=1)$ levels. Since the first term gives rise to the removal of degeneracy expected from group theoretical considerations, we did not include the second term in our numerical evaluations. This procedure was sufficiently accurate to provide a useful check on the validity of the more accurate numerical solutions for the threecomponent wave functions of our set of mesh points. In Table VII these numerical results ("full mesh") are given and are compared to the results using perturbation theory, as in Eq. (18). As can be seen, the two approaches yield quite compatible results.

\section{Qualitative Remarks}

Some additional comments on Eq. (18) are in order. The first line of this equation gives the energy at first order in perturbation theory. At this order the wave function remains a product of the spatial ground-state spatial wave function for a $J=0$ molecule times a $J=1$

rotational wave function. At this level of approximation there is no dynamical coupling between translation and rotation. In second order perturbation theory we see that admixtures of two phonon states which are multiplied by different rotational states are introduced. For example, consider the situation when the molecule is in a uniaxial symmetry site and let $|X\rangle,|Y\rangle$, and $|Z\rangle$ be the $J=1$ states for which respectively $J_{x}, J_{y}$, and $J_{z}$ are zero. If, for simplicity, we 
assume that the unperturbed spatial wave function is spherically symmetric, then the state which without perturbation was

$$
C_{0}|Z\rangle e^{-r^{2} /\left(4 \sigma^{2}\right)}
$$

where $C_{0}$ is a normalization constant, is now

$$
C_{1}|Z\rangle e^{-r^{2} / 4 \sigma^{2}}+C_{2}|X\rangle z x e^{-r^{2} /\left(4 \sigma^{2}\right)}+C_{2}|Y\rangle z y e^{-r^{2} /\left(4 \sigma^{2}\right)},
$$

where $C_{1} \approx C_{0}$ and $C_{2}$ is small compared to $C_{1}$. The point is that this formulation allows the molecule to change its orientational state as it translates. For $\mathrm{H}_{2}$ in $\mathrm{C}_{60}$ this effect is small, however, in less symmetrical cavities, as in nanotubes ${ }^{16}$, this effect can become more important.

\section{Group Theoretical Analysis}

In Fig. 2 we show the influence of roton-phonon coupling and local site symmetry on the energy spectrum of the one phonon- $(J=1)$ manifold. At the far left we start from the case of highest symmetry when the phonon and rotations separately have complete rotational invariance and no phonon-roton coupling is present. In this case the manifold of 9 states (3 one-phonon states $\otimes 3(J=1)$ states $)$ is completely degenerate. When roton-phonon cou-

pling is included (but the environment is still spherically symmetric) we have overall rotational invariance and the resulting eigenstates are characterized by their total angular momentum $K$. The roton-phonon coupling causes states with different $K$ to have different energy. (The size of the splitting shown in the figure is adjusted to agree with the center of gravity of the appropriate levels for cubic site symmetry.)

The two right-hand columns pertain to the situation when a $(J=1)$ hydrogen molecule occupies the octahedral interstitial site of $\mathrm{C}_{60}$. When the $\mathrm{C}_{60}$ molecules are orientationally disordered the interstitial site has $\mathrm{O}_{h}$ symmetry and we consider that case first. Use of the character tables for the $\mathrm{O}_{\mathrm{h}}$ group indicates that the original 9 dimensional reducible representation $\Gamma$ is decomposed into irreducible representation of the $\mathrm{O}_{\mathrm{h}}$ group as

$$
\Gamma=T_{2 \mathrm{~g}} \oplus T_{1 \mathrm{~g}} \oplus E_{\mathrm{g}} \oplus A_{\mathrm{g}} .
$$


and the basis functions associated with these irreducible representations are given in Table VIII. As mentioned above, for temperatures below about $260 \mathrm{~K}$, the $\mathrm{C}_{60}$ molecules order into a structure of crystal symmetry $\mathrm{Pa}^{9}{ }^{-12}$ in which case the formerly octahedral interstitial has the lower $\mathrm{S}_{6}$ symmetry. ${ }^{13}$ Use of the relevant character table shows that now

$$
\Gamma=3 A_{\mathrm{g}} \oplus 3 E_{\mathrm{g}} \oplus 3 E_{\mathrm{g}}^{*},
$$

where $E_{g}$ is a complex one dimensional representation and $E_{g}^{*}$ is its complex conjugate partner. The basis functions associated with these irreducible representations are given in Table VIII. The most important conclusion from this analysis is that the energy eigenfunctions are not simply products of translational and rotation wave functions, but instead are linear combinations of such products. This type of wavefunction reflects the fact that symmetry operations act simultaneously on the position and the orientation of a molecule.

To emphasize this fact we give, in Fig. 3, a pictorial representations of the translationrotation wavefunctions. This representation is to be interpreted as follows. We know that the rotational wave functions for a free $J=1$ molecule can be taken to be analogs of $p_{x}, p_{y}$ and $p_{z}$ functions and we will label these rotational wavefunctions as $X, Y$, or $Z$. For instance $|X\rangle \sim \sin \theta \cos \phi,|Y\rangle \sim \sin \theta \sin \phi$, and $|Z\rangle \sim \cos \theta$. These wave functions have two lobes, one positive the other negative aligned along the axis associated with their state label. When such a rotational function is multiplied by a one phonon function in the $\alpha$ direction, $(|x\rangle$ denotes a wave function for a single phonon excitation in the $x$-direction), the total wave function will be an odd function of $\alpha$. Thus the wave function $|x X\rangle$ is an odd function of $x$ and is therefore depicted by two $p_{x}$ functions, one at positive $x$ and another at $-x$ with the signs of the two lobes changed. For simplicity in the figures we show only those functions which have appropriate dependence in the plane of the paper, which is taken to be the $x-y$ plane. At the upper left we show an $x y$-like function. It has two other $t_{2 g}$ symmetry partners which are $x z$-like and $y z$-like. At the upper right we show one of the two $\mathrm{E}_{g}$ functions which is $x^{2}-y^{2}$-like. These five $\mathrm{t}_{2 g}$ and $\mathrm{E}_{g}$ functions comprise the manifold of total angular momentum $K=2$ states. Within spherical symmetry all five of these states are degenerate in energy. In the lower left of Fig. 3 we show the $z$-like function of $t_{1 g}$ symmetry. Its other two partners are obtained by cyclically permuting $x, y$, and $z$. These three functions comprise the manifold of total angular 
momentum $K=1$ states, which transform under rotation as a vector. Finally at the lower right we show the angular momentum $K=0$ state. Thus in spherical symmetry, the nine $J=1$ single phonon states give rise to three distinct energy manifolds which have degeneracies 1,3 , and 5, corresponding respectively to total angular momentum $K=0, K=1$, and $K=2$.

The simplest classical arguments do not reproduce the above results. For instance, one might argue that translation can occur equivalently along either of three equivalent coordinate axes. In each case, one can have the molecule oriented along the axis of translational motion or perpendicular to that axis. This argument would suggest that the nine levels break into a three-fold degenerate energy level in which the molecule is oriented longitudinally and a six-fold level in which the molecule is oriented transversely. This discussion shows that it is essential to treat the translation-rotation problem quantum mechanically to get the correct degeneracy.

\section{NEUTRON SPECTRUM}

In the experimental study of Fitzgerald et. al. ${ }^{5}$, neutron energy loss spectrum of $\mathrm{H}_{2}$ trapped in $\mathrm{C}_{60}$ was measured with an energy resolution of $0.3 \mathrm{meV}$. The spectrum shows surprisingly rich features. However the origin of these features were not successfully identified in detail. Since the observed neutron spectrum is a direct probe of the intermolecular potential between $\mathrm{H}_{2}$ molecules and $\mathrm{C}_{60}$ host lattice, it is very important to see if available atom-atom potentials can give a spectrum which is similar to the experimental data. A suitable analysis of the high resolution inelastic neutron scattering data in Ref. 5 should, in principle, give a detailed information about the intermolecular potential between $\mathrm{H}_{2}$ molecules and the host lattice.

Figure 4 illustrates several possible transitions, involving both rotational and vibrational excitations, that could be observed in a neutron scattering experiment for $\mathrm{H}_{2}$ in solid $\mathrm{C}_{60}$. In order to estimate the intensities of these transitions and the corresponding neutron spectrum, in Appendix $\mathrm{C}$ we derive the inelastic neutron cross section for trapped $\mathrm{H}_{2}$ molecules in a powder

sample at low temperature. Below we discuss the contribution to the total neutron spectrum from each of these transitions, labeled as $T_{A}, \ldots, T_{E}$, and then compare the calculated spectrum with experimental data using various atom-atom potentials.

We start with the transitions involving phonon creation in para hydrogen, as shown by 
$T_{D}$ in Fig.4. Because of the spin-dependent interaction between the proton and the neutron, processes in which a para molecule is not converted to an ortho molecule are forbidden, or more correctly speaking, are proportional to the coherent cross section $b$, which is very small compared to incoherent cross section $b^{\prime}$. Hence, the transition $T_{D}$ will not have a noticeable contribution to the total cross section.

We next discuss the contribution to the total spectrum from processes in which either a $(J=0)$ molecule is converted to $(J=1)$ molecule (para-ortho conversion, labeled as $T_{A}$ in Fig. 4) or a single phonon is created (as shown by $T_{B}$ ). Our calculations presented so far indicate that both processes will give features around $14 \mathrm{meV}$ in neutron spectrum.

In the Appendix $\mathrm{C}$ we find that the cross section due to para-ortho conversion, (indicated by the subscript $0 \rightarrow 1$ is given by

$$
\left.\frac{\partial^{2} \sigma}{\partial \Omega \partial E}\right)_{0 \rightarrow 1}=\frac{3}{4} N \frac{k^{\prime}}{k}(1-x)\left[b^{\prime} j_{1}\left(\frac{1}{2} \kappa \rho\right)\right]^{2} e^{-2 W(\kappa)} \sum_{m} \delta\left[E_{L}-\left(E_{c}+E_{m}\right)\right],
$$

where $N$ is the total number of $\mathrm{H}_{2}$ molecules, $\mathbf{k}\left(\mathbf{k}^{\prime}\right)$ is wave vector of the incident (scattered) neutron, $\boldsymbol{\kappa}=\mathbf{k}^{\prime}-\mathbf{k}, x$ is the fraction of $\mathrm{H}_{2}$ molecules which are ortho (odd $J$ ) molecules, $\rho$ is the separation between protons in the $\mathrm{H}_{2}$ molecule, $b^{\prime}$ is the spin-dependent cross section in the proton-neutron pseudopotential, $j_{n}$ is the $n$th order spherical Bessel function, and $W(\kappa)$ is the Debye-Waller factor which we take to be $\frac{1}{3} \kappa^{2}\left\langle u^{2}\right\rangle$. Also, $E_{L}$ is the energy loss of the neutron, and $E_{c}+E_{m}$ is the para-ortho conversion energy when the final state of the ortho has $J_{z}=m$.

Similarly, the cross section due to ortho-para conversion, $\left.\frac{\partial^{2} \sigma}{\partial \Omega \partial E}\right)_{1 \rightarrow 0}$ has the same expression as Eq. (23) but now the factor $(1-x)$ is replaced by $x$. Hence the ratio of the total cross section for ortho to para conversion to that of para to ortho conversion is $(1-x)$ to $x$, where $x$ is the ortho concentration. Normally the ratio of energy gain to energy loss cross sections follows the Boltzmann factor. Here, the populations are set by $x$ rather than by the temperature.

We now discuss the cross section due to phonon creation on a $(J=1)$ molecule (indicated here by the subscript $1 \rightarrow 1$ ), which is calculated in the Appendix C. These transitions are shown as $T_{B}$ in Fig. 4. The result requires a knowledge of the translation-rotation wave function of the $\mathrm{H}_{2}$ molecule. We find that

$$
\left.\frac{\partial^{2} \sigma}{\partial \Omega \partial E}\right)_{1 \rightarrow 1}=N x \frac{k^{\prime}}{k} b^{\prime 2} \sum_{n=1}^{4} S_{1 \rightarrow 1, n}^{(1)}
$$


where the cross section $S_{1 \rightarrow 1, n}^{(1)}$ are given in Eqs. (C34), (C35), and (C43) of Appendix C.

In Fig. $5 \mathrm{~T}_{\mathrm{B}}$ represents the transitions from the $(J=1, N=0)$ levels to the manifold of nine energy levels of $(J=1, N=1)$. Accordingly, we expect several transitions with nonzero amplitude and thus rich features in the total neutron cross section.

Also one may consider the cross section integrated over energy, which is a usefull quantity to indicate the relative strength of the different transitions discussed above. The ratio, $r_{P}$, of the integrated neutron energy-loss cross section for phonon creation (process $\mathrm{T}_{\mathrm{B}}$ in Fig. divided by that for para-ortho conversion (process $\mathrm{T}_{\mathrm{A}}$ in Fig. 4) was found in the Appendix $\mathrm{C}$ to be

$$
r_{P}=\frac{2}{27} \kappa^{2}\left\langle u^{2}\right\rangle \frac{x\left[j_{0}\left(\frac{1}{2} \kappa \rho\right)^{2}+2 j_{2}\left(\frac{1}{2} \kappa \rho\right)^{2}\right]}{(1-x) j_{1}\left(\frac{1}{2} \kappa \rho\right)^{2}} .
$$

This ratio is plotted as a function of $\kappa$ for $x=3 / 4$ in Fig. 6. Since this ratio is of order unity, the energy loss spectrum will display features due to both phonons and para-ortho conversion.

In appendix $\mathrm{C}$ we also calculate the zero phonon ortho cross section for the transition shown as $T_{E}$ in Fig. 4. Since the $(J=1)$ levels are in thermal equilibrium, in this case the ratio of the cross sections at energy gain to those of energy loss does satisfy detailed balance. The ratio of the total cross section (counting both energy loss and energy gain) for transitions within the $(J=1)$ ground manifold to that due to para to ortho conversion was found at zero temperature to be

$$
r_{J=1}(T=0)=\frac{x}{1-x} \frac{4 j_{2}\left(\frac{1}{2} \kappa \rho\right)^{2}}{15 j_{1}\left(\frac{1}{2} \kappa \rho\right)^{2}} .
$$

Figure 6 shows that this ratio is quite small and therefore experimental observation of this transition (i.e. $T_{E}$ ) would be very difficult.

Figure 7 shows the neutron energy loss spectrum and the calculated total spectrum using the same potential, so called WS77 model, ${ }^{15}$ used by FitzGerald et. $a l^{5}$. Even though the calculated spectum is wider than the experimental spectrum, it is still possible to make a one to one correspondence between calculation and experiment as is shown by arrows in Fig. 7. The top curve in this figure shows what the spectrum looks like if the orientational part of the potential is scaled by about half. The agreement between the data and calculations is somewhat better after this arbitrary scaling, indicating that the potential used is too anisotropic for the 
center of mass motion of $\mathrm{H}_{2}$ molecule but at the same time it is too weak for the orientational part of $\mathrm{H}_{2}$ (because it gives too small a result for $\delta$, the splitting of the $(J=1)$ ground manifold).

We also tested other potentials commonly used in the literature and these results are shown in Fig. 8. The top curve is from Novaco's 6-12 potential which was developed to study hydrogen on graphite. ${ }^{4}$ Clearly this potential gives too low phonon energies and too little splitting of the $(J=1)$ levels for $\mathrm{H}_{2}$ in solid $\mathrm{C}_{60}$. The other two curves in Fig. 8 are 6-exp potentials tabulated in Ref. 15. The spectrum from these potentials does not agree with experiment either. We also searched the potential parameters $A$ and $B$ for 6-12 and $A, B$, and $C$ for 6 -exp types of potentials. However we were not able to improve the fit to experiment using these atom-atom potentials. Hence, it seems that simple atom-atom potential does not describe the details of the $\mathrm{H}_{2}-\mathrm{C}_{60}$ interaction well. It is an open and important question to find a better potential can reproduce the experimental spectrum better. It is also of important to see how well the potentials obtained from density functional theory within local density approximation will do.

Finally, in addition to features observed near $14 \mathrm{meV}$, Fitzgerald et al. ${ }^{5}$ also observed a feature at about $28 \mathrm{meV}$ in the energy gain time-of-flight spectrum. This energy is about twice that of either the para-ortho conversion energy or the energy of the translational phonon for an $\mathrm{H}_{2}$ molecule in an octahedral interstitial site. Clearly this feature represents the energy of two excitations, but it was not clear whether these would be two phonons, one phonon and one ortho-para transition or two ortho-para transitions. In Fig. 9 we show the temperature dependence of the total intensity of this feature. This temperature dependence follows a thermal activation with an energy of about $14 \mathrm{meV}$. Thus the initial state must consist of one thermally excited phonon and the transition observed destroys one thermal phonon and converts one ortho molecule (which occurs with temperature-independent probability $x$ ) to a para molecule, thus giving the observed energy of about $28 \mathrm{meV}$.

\section{CONCLUSIONS}

Our results indicate that the coupled phonon-roton problem is a rich one. For the light molecules of $\mathrm{H}_{2}$ where the splitting of rotational levels is large compared to most lattice vibra- 
tional modes, one is in a so-called weak-coupling limit where the interactions between rotons and translational phonons can be treated perturbatively. Even at this level one recovers an interesting structure.

Needless to say, we hope that the calculations in this paper will motivate more detailed experiments at higher resolution to elucidate the structure of the roton-phonon spectrum.

We may summarize our main conclusions as follows:

- We have presented a systematic perturbative approach to the calculation of the rotonphonon spectrum of hydrogen molecules in confined geometry. Our calculations agree with the group theoretical analysis for the geometries considered here.

- In a general way, the techniques of this paper (use of the atom-atom potential combined with perturbation theory) may prove useful to treat hydrogen molecules in other confined geometries, in particular in or on nanotubes. We are currently analyzing this situation.

- We give the first calculation of the expected energy loss spectrum from hydrogen in $\mathrm{C}_{60}$ in the energy range where phonons and para-ortho conversion both are important. We find that none of the traditional 6-12 and 6-exp types of potentials give good results for the detailed energy dependence of the observed phonon spectrum, although the WS77 potential ${ }^{15}$ we used was definitely the most satisfactory. It is a theoretical challenge to determine a potential which fully reproduces the observed spectrum.

- We identify the feature at $28 \mathrm{meV}$ in the energy gain spectrum as consisting of conversion of one ortho molecule to a para molecule combined with annihilation of a single phonon. This identification is uniquely indicated by the temperature dependence of this feature.

\section{ACKNOWLEDGEMENT}

We acknowledge partial support from the Israel-US Binational Science Foundation. 


\section{APPENDIX A: ATOM-ATOM POTENTIAL AND $A_{L}^{M}$}

We model the interactions between the hydrogen molecule and the surrounding cage of $\mathrm{C}_{60}$ molecules using an atom-atom potential. ${ }^{15}$ Unless otherwise indicated, all the results reported in this paper are obtained from the same potential $-A / r^{6}+B \exp (-C r)$ that is used in Ref. 5 (where $A=5.94 \mathrm{eV} \AA^{6}, B=678.2 \mathrm{eV}$, and $C=3.67 \AA^{-1}$ ).

We consider two cases depending on the orientational state of $\mathrm{C}_{60}$ molecules. When the molecules in the surrounding cage are orientationally disordered, we distribute the carbon centers uniformly over the surface of a sphere. This case corresponds to the octahedral symmetry discussed in the text. The resulting integration of the atom-atom potential over a spherical surface is done analytically in Ref. 5 and therefore is not given here. For the Pa $\overline{3}$ symmetry, the $\mathrm{C}_{60}$ molecules are rotated according to their Pa $\overline{3}$ settings and then the total potential and $A_{l}^{m}$ 's are calculated on a mesh points of a cube centered at the octahedral site. Below we derive a convenient way to obtain the $A_{l}^{m}$ 's from the atom-atom potential.

We write the potential, $V_{\mathrm{H}-\mathrm{C}}$ between a single $\mathrm{H}$ atom and a single $\mathrm{C}$ atom as

$$
V_{\mathrm{H}-\mathrm{C}}=F(r)
$$

where $\mathbf{r}$ is the displacement of the $\mathrm{C}$ atom relative to to the $\mathrm{H}$ atom. Thus the interaction, $V\left(\mathrm{H}_{2}\right)$ of a $\mathrm{H}_{2}$ molecule with a $\mathrm{C}$ atom can be written as

$$
V\left(\mathrm{H}_{2}\right)=\sum_{\sigma= \pm 1} F\left(\left[r^{2}+\frac{1}{4} \rho^{2}+\sigma \rho \mathbf{r} \cdot \hat{n}\right]^{1 / 2}\right)
$$

where now $\mathbf{r}$ is the displacement of the $\mathrm{C}$ atom relative to the center of the $\mathrm{H}_{2}$ molecule whose atoms are at positions $\pm \frac{1}{2} \rho \hat{n}$, where $\hat{n}$ is a unit vector specifying the orientation of the molecular axis of the $\mathrm{H}_{2}$ molecule. Then

$$
A_{2 l}^{m}=\sum_{\sigma} \sum_{i} \int Y_{2 l}^{m}(\hat{n})^{*} F\left(\left[r_{i}^{2}+\frac{1}{4} \rho^{2}+\sigma \rho \mathbf{r}_{i} \cdot \hat{n}\right]^{1 / 2}\right) d \Omega
$$

where $d \Omega$ indicates an integration over all orientations of $\hat{n}$ and the sum over $i$ is over all neighboring carbon atoms. We use this to get

$$
A_{0}^{0} \equiv V_{0}=2 \sqrt{\pi} \sum_{i} \int_{-1}^{1} F\left(\left[r_{i}^{2}+\frac{1}{4} \rho^{2}+\rho r_{i} x\right]^{1 / 2}\right) d x
$$


To get the $A_{l}^{m}$ 's for $l>0$ we write

$$
\sum_{\sigma} F\left(\left[r_{i}^{2}+\frac{1}{4} \rho^{2}+\sigma \rho \mathbf{r}_{i} \cdot \hat{n}\right]^{1 / 2}\right)=\sum_{L} B_{2 L}\left(r_{i}\right) Y_{2 L}^{0}\left(\theta_{r, n}\right)
$$

where $\theta_{r, n}$ is the angle between the vectors $\mathbf{r}_{i}$ and $\hat{n}$. We have that

$$
B_{2 L}\left(r_{i}\right)=2 \pi \sum_{\sigma} \int_{0}^{\pi} F\left(\left[r_{i}^{2}+\frac{1}{4} \rho^{2}+\sigma \rho \mathbf{r}_{i} \cos \theta\right]^{1 / 2}\right) Y_{2 L}^{0}(\theta) \sin \theta d \theta
$$

Now substitute Eq. (A5) into Eq. (A3) to get

$$
A_{2 l}^{m}=\sum_{i} \int Y_{2 l}^{m}(\hat{n})^{*} \sum_{L} B_{2 L}\left(r_{i}\right) Y_{2 L}^{0}\left(\theta_{r, n}\right) d \Omega
$$

Using the addition theorem for spherical harmonics ${ }^{24}$ we have

$$
\begin{aligned}
A_{2 l}^{m} & =\sum_{i} \int Y_{2 l}^{m}(\hat{n})^{*} \sum_{L} B_{2 L}\left(r_{i}\right) \sqrt{4 \pi /(2 L+1)} \sum_{\nu} Y_{2 L}^{\nu}(\hat{n}) Y_{2 L}^{\nu}\left(\hat{\mathbf{r}}_{i}\right)^{*} d \Omega \\
& =\sum_{i} B_{2 l}\left(r_{i}\right) \sqrt{4 \pi /(2 l+1)} Y_{2 l}^{m}\left(\hat{\mathbf{r}}_{i}\right)^{*}
\end{aligned}
$$

For $A_{2}^{m}$ we get

$$
A_{2}^{m}=2 \pi \sum_{i} Y_{2}^{m}\left(\hat{\mathbf{r}}_{i}\right)^{*} \int_{-1}^{1}\left(3 x^{2}-1\right) F\left(\left[r_{i}^{2}+\frac{1}{4} \rho^{2}+r_{i} \rho x\right]^{1 / 2}\right) d x .
$$

For power-law functions $F$ (i.e. $F \sim 1 / r^{2 n}$ ), this integral can be done analytically.

\section{APPENDIX B: SPHERICAL CAVITY}

In this appendix we apply our formalism discussed in the text to a simple toy model to utilize the main physics of quantum roton-phonon dynamics of a $\mathrm{H}_{2}$ molecule confined in a spherically symmetric cavity for which the potential differs perturbatively from harmonic.

\section{Orientationally Dependent Potential}

For a diatomic molecule for which the spherical part of the potential is that of an isotropic spherical oscillator, we take the orientationally dependent part of the potential to be

$$
U(\mathbf{r}, \Omega)=f(r)\left[(\hat{x} \sin \theta \cos \phi+\hat{y} \sin \theta \sin \phi+\hat{z} \cos \theta)^{2}-\frac{1}{3}\right]
$$


where $\hat{x} \equiv x / r, \hat{y} \equiv y / r$, and $\hat{z}=z / r$.

This potential can be written in the canonical form of Eq. (2) with

$$
A_{2}^{m}=\frac{8 \pi}{15} f(r) Y_{2}^{m}(\hat{r})^{*}
$$

\section{Energy Shift of the $J=1$ Manifold}

To evaluate Eq. (16) for the shift in the center of gravity of the $J=1$ zero phonon levels, we need the wave function of the ground state, namely

$$
\psi_{0}(\mathbf{r})=\alpha e^{-r^{2} /\left(4 \sigma^{2}\right)}
$$

where $\alpha=\sigma^{-3 / 2}(2 \pi)^{-3 / 4}$. Also we can write the two phonon excited states (for spherical symmetry) as

$$
\psi_{2, m}^{(L=2)}=\beta(r / \sigma)^{2} Y_{2}^{m}(\hat{\mathbf{r}}) e^{-r^{2} /\left(4 \sigma^{2}\right)}
$$

for $m=-2,-1,0,1,2$ and for angular momentum $L=2$ and where $\beta=\sigma^{-3 / 2} \sqrt{2 / 15}(2 \pi)^{-1 / 4}$. The sixth two-phonon state is an $s$-wave state, whose wave function we do not need. We will also need the $L=2$ (d-wave) four phonon states which are

$$
\psi_{4, m}^{(L=2)}=\gamma\left[(r / \sigma)^{4}-7(r / \sigma)^{2}\right] Y_{2}^{m}(\hat{\mathbf{r}}) e^{-r^{2} /\left(4 \sigma^{2}\right)},
$$

for $m=-2,-1,0,1,2$ and for angular momentum $L=2$ and where $\gamma=\sigma^{-3 / 2} \sqrt{1 / 105}(2 \pi)^{-1 / 4}$.

Now we assume the following polynomial fit for $f(r)$ :

$$
f(r)=\gamma_{2}(r / \sigma)^{2}+\gamma_{4}(r / \sigma)^{4}+\gamma_{6}(r / \sigma)^{6}
$$

We then find

$$
\left|\left\langle 0\left|A_{2}^{m}(\mathbf{r})\right| \psi_{2, m}^{(L=2)}\right\rangle\right|=4 \sqrt{\frac{\pi}{15}}\left|\gamma_{2}+7 \gamma_{4}+63 \gamma_{6}\right| \equiv 4 \sqrt{\frac{\pi}{15}} \Gamma
$$

and

$$
\left|\left\langle 0\left|A_{2}^{m}(\mathbf{r})\right| \psi_{4, m}^{(L=2)}\right\rangle\right|=4 \sqrt{\frac{14 \pi}{15}}\left|\gamma_{4}+18 \gamma_{6}\right|
$$


Thus we have

$$
\Delta E=-\frac{4}{15} \frac{\left(\gamma_{2}+7 \gamma_{4}+63 \gamma_{6}\right)^{2}}{\hbar \omega}-\frac{28}{15} \frac{\left(\gamma_{4}+18 \gamma_{6}\right)^{2}}{\hbar \omega}
$$

From numerics we get

$$
A_{2}^{0}(z)=0.07226 r^{4}+0.0348 r^{6}
$$

For convenience we take $\sigma=0.25 \AA$. Then $A_{2}^{0}$ is of the form of Eq. (B6) with

$$
\begin{gathered}
\gamma_{4}=0.07226 \sigma^{4}, \quad \gamma_{6}=0.0348 \sigma^{6} . \\
\gamma_{4}=0.07226 / 256=0.282 \mathrm{meV} \\
\gamma_{6}=0.0348 / 4096=0.0084 \mathrm{meV} .
\end{gathered}
$$

we evaluate the first and second terms on the right-hand side of Eq. (B9) to be $0.115 \mathrm{meV}$ and $0.025 \mathrm{meV}$, respectively. Thus we have

$$
\Delta E=0.14 \mathrm{meV}
$$

Notice that $\Delta E$ is a very strong function of $\sigma$. For instance, if you take $\sigma=0.26 \AA$, you get $\gamma_{4}=0.33 \mathrm{meV}$ and $\gamma_{6}=0.011 \mathrm{meV}$ in which case $\Delta E=0.19 \mathrm{meV}$.

\section{Effect of Translation-Rotation Coupling on the $J=1$ One Phonon Manifold}

In the absence of coupling between translations and rotations we characterize the singlephonon states by phonon angular momentum $L_{P}$, so that

$$
\left|L_{P}= \pm 1\right\rangle=\mp\left(\frac{x \pm i y}{\sigma}\right) \psi_{0}(\mathbf{r}), \quad\left|L_{P}=0\right\rangle=(z / \sigma) \psi_{0}(\mathbf{r}) .
$$

We now wish to include the effect of the perturbation of the form of Eq. (2) when the coefficients are as in Eq. (B2), with $f(r)$ given by Eq. (B6). We know that states are now characterized by the total angular momentum $\mathbf{K}=\mathbf{L}_{\mathbf{P}}+\mathbf{J}$. So the energy of the $K=2$ manifold is given by 


$$
\begin{aligned}
E(K=2) & =\left\langle L_{P}=1, J_{z}=1|U(\mathbf{r}, \Omega)| L_{P}=1, J_{z}=1\right\rangle \\
& =-\frac{1}{15} \frac{\int d \mathbf{r}\left|\frac{x-i y}{\sigma}\right|^{2} e^{-r^{2} /\left(2 \sigma^{2}\right)} f(r) \frac{3 z^{2}-r^{2}}{r^{2}}}{\int d \mathbf{r}\left|\frac{x-i y}{\sigma}\right|^{2} e^{-r^{2} /\left(2 \sigma^{2}\right)}}\left\langle J_{z}=1\left|\left(3 J_{z}^{2}-2\right)\right| J_{z}=1\right\rangle \\
& =\frac{2 \int d \mathbf{r} r^{2} f(r) e^{-r^{2} /\left(2 \sigma^{2}\right)}}{225 \sigma^{2} \int d \mathbf{r} e^{-r^{2} /\left(2 \sigma^{2}\right)}}=\frac{2}{15} \Gamma,
\end{aligned}
$$

where $f(r)$ and $\Gamma$ are defined in Eq. (B6) and Eq. (B7), respectively.

Similarly, one can evaluate the Hamiltonian in the manifold of states $\phi_{1}=\left|L_{P}=1, J_{z}=0\right\rangle$ and $\phi_{2}=\left|L_{P}=0, J_{z}=1\right\rangle$. The Hamiltonian matrix in this basis is found to be

$$
\mathcal{H}=\Gamma\left[\begin{array}{cc}
-\frac{4}{15} & \frac{2}{5} \\
\frac{2}{5} & -\frac{4}{15}
\end{array}\right] \text {. }
$$

This matrix has an eigenvalue $\frac{2}{15} \Gamma$ which is associated with the $K=2, K_{z}=1$ state and the new eigenvalue for the $K=1$ manifold, $E(K=1)=-\frac{2}{3} \Gamma$.

Similarly, one can evaluate the Hamiltonian in the manifold of states $\phi_{1}=\mid L_{P}=1, J_{z}=$ $-1\rangle, \phi_{2}=\left|L_{P}=0, J_{z}=0\right\rangle$ and $\phi_{3}=\left|L_{P}=-1, J_{z}=1\right\rangle$. The Hamiltonian matrix in this basis is found to be

$$
\mathcal{H}=\Gamma\left[\begin{array}{ccc}
\frac{2}{15} & -\frac{2}{5} & \frac{4}{5} \\
-\frac{2}{5} & \frac{8}{15} & -\frac{2}{5} \\
\frac{4}{5} & -\frac{2}{5} & \frac{2}{15}
\end{array}\right]
$$

In this manifold we reproduce the eigenvalues for $K=2$ and $K=1$. The new eigenvalue is $E(K=0)=\frac{4}{3} \Gamma$.

\section{APPENDIX C: NEUTRON SCATTERING CROSS SECTION}

\section{GENERAL FORMULATION}

Following Elliott and Hartmann ${ }^{23}$, we write

$$
V\left(\mathbf{r}-\mathbf{r}_{n}\right)=\frac{2 \pi \hbar}{m_{0}} \delta\left(\mathbf{r}-\mathbf{r}_{n}\right)\left[b+b^{\prime}(\boldsymbol{\sigma} \cdot \mathbf{I})\right],
$$

where $m_{0}$ is the neutron mass, $\boldsymbol{\sigma}$ is the neutron spin, $\mathbf{r}$ is the coordinate of the proton, $\mathbf{r}_{n}$ the coordinate of the neutron, $I$ is the proton spin, and $b$ and $b^{\prime}$ are the coherent and incoherent 
scattering lengths for this scattering. Since $b$ is very small, we drop that term from now on. The differential scattering cross section is

$$
\frac{\partial^{2} \sigma}{\partial \Omega \partial E}=\frac{k^{\prime}}{k} \sum_{i f} P_{i} \delta\left(E-E_{i}+E_{f}\right)|V|^{2},
$$

where $E=\hbar^{2}\left[\left(k^{\prime}\right)^{2}-k^{2}\right] /\left(2 m_{0}\right)$ and the sum is over all states of the system. Here the potential is

$$
V=b^{\prime} \sum_{j \alpha} e^{i \boldsymbol{\kappa} \cdot \mathbf{R}_{j, \alpha}} \boldsymbol{\sigma} \cdot I_{j, \alpha},
$$

where $\boldsymbol{\kappa}=\mathbf{k}^{\prime}-\mathbf{k}, j$ labels molecules and $\alpha=1,2$ the atoms within a molecule. Performing the sum over $\alpha$ we get

$$
V=b^{\prime} \sum_{j} e^{i \boldsymbol{\kappa} \cdot \mathbf{R}_{j}}\left(\left(\boldsymbol{\sigma} \cdot I_{j}\right) \cos \left(\frac{1}{2} \boldsymbol{\kappa} \cdot \boldsymbol{\rho}\right)+i \sin \left(\frac{1}{2} \boldsymbol{\kappa} \cdot \boldsymbol{\rho}\right)\left[\boldsymbol{\sigma} \cdot\left(\mathbf{I}_{j 1}-\mathbf{I}_{j 2}\right)\right]\right),
$$

where $\mathbf{I}_{j}=\mathbf{I}_{j 1}+\mathbf{I}_{j 2}$. The first term acts only on ortho molecules because for paras the total spin is zero and the second term causes transitions between ortho and para molecules. So we write the scattering cross section as the sum of three terms, the first of which represents scattering from an ortho molecule and others ortho-para conversion or the reverse. Thus

$$
\frac{\partial^{2} \sigma}{\partial \Omega \partial E}=\frac{k^{\prime}}{k}\left[N x \mathcal{S}_{1 \rightarrow 1}+N x \mathcal{S}_{1 \rightarrow 0}+N(1-x) \mathcal{S}_{0 \rightarrow 1}\right]
$$

where $N$ is the total number of molecules and $x$ is the ortho concentration. Because there are no correlations between nuclear spins each cross section is actually a sum over cross sections for each molecule:

$$
\mathcal{S}_{\beta}=\sum_{j} \mathcal{S}_{\beta j}
$$

where $j$ labels the molecule, $\beta$ is $0 \rightarrow 1$, etc., and

$$
\begin{aligned}
& \mathcal{S}_{0 \rightarrow 1, j}=\sum_{J_{i}=0, J_{f}=1} P_{i} \delta\left(E-E_{i}+E_{f}\right)\left|\left\langle f\left|b^{\prime} e^{i \boldsymbol{\kappa} \cdot \mathbf{R}_{j}} \sin \left(\frac{1}{2} \boldsymbol{\kappa} \cdot \boldsymbol{\rho}\right)\left[\boldsymbol{\sigma} \cdot\left(\mathbf{I}_{j 1}-\mathbf{I}_{j 2}\right)\right]\right| i\right\rangle_{T}\right|^{2} \\
& \mathcal{S}_{1 \rightarrow 0, j}=\sum_{J_{i}=1, J_{f}=0} P_{i} \delta\left(E-E_{i}+E_{f}\right)\left|\left\langle f\left|b^{\prime} e^{i \boldsymbol{\kappa} \cdot \mathbf{R}_{j}} \sin \left(\frac{1}{2} \boldsymbol{\kappa} \cdot \boldsymbol{\rho}\right)\left[\boldsymbol{\sigma} \cdot\left(\mathbf{I}_{j 1}-\mathbf{I}_{j 2}\right)\right]\right| i\right\rangle_{T}\right|^{2} \\
& \mathcal{S}_{1 \rightarrow 1, j}=\sum_{J_{i}=1, J_{f}=1} P_{i} \delta\left(E-E_{i}+E_{f}\right)\left|\left\langle f\left|b^{\prime} e^{i \boldsymbol{\kappa} \cdot \mathbf{R}_{j}} \cos \left(\frac{1}{2} \boldsymbol{\kappa} \cdot \boldsymbol{\rho}\right)[\boldsymbol{\sigma} \cdot \mathbf{I}]\right| i\right\rangle_{T}\right|^{2},
\end{aligned}
$$


where the sums are over states for the fixed species (ortho or para) of molecule as indicated and the subscript $\mathrm{T}$ indicates that the wavefunctions include nuclear spin functions.

Now we perform the sum over the spin states of the neutron and proton to obtain the results

$$
\begin{aligned}
& \mathcal{S}_{0 \rightarrow 1, j}=\frac{3}{4}\left(b^{\prime}\right)^{2} \sum_{J_{i}=0, J_{f}=1} P_{i} \delta\left(E-E_{i}+E_{f}\right)\left|\left\langle f\left|e^{i \boldsymbol{\kappa} \cdot \mathbf{R}_{j}} \sin \left(\frac{1}{2} \boldsymbol{\kappa} \cdot \boldsymbol{\rho}\right)\right| i\right\rangle\right|^{2} \\
& \mathcal{S}_{1 \rightarrow 0, j}=\frac{1}{4}\left(b^{\prime}\right)^{2} \sum_{J_{i}=1, J_{f}=0} P_{i} \delta\left(E-E_{i}+E_{f}\right)\left|\left\langle f\left|e^{i \boldsymbol{\kappa} \cdot \mathbf{R}_{j}} \sin \left(\frac{1}{2} \boldsymbol{\kappa} \cdot \boldsymbol{\rho}\right)\right| i\right\rangle\right|^{2} \\
& \mathcal{S}_{1 \rightarrow 1, j}=\frac{1}{2}\left(b^{\prime}\right)^{2} \sum_{J_{i}=1, J_{f}=1} P_{i} \delta\left(E-E_{i}+E_{f}\right)\left|\left\langle f\left|e^{i \boldsymbol{\kappa} \cdot \mathbf{R}_{j}} \cos \left(\frac{1}{2} \boldsymbol{\kappa} \cdot \boldsymbol{\rho}\right)\right| i\right\rangle\right|^{2},
\end{aligned}
$$

where now states $|f\rangle$ and $|i\rangle$ no longer include nuclear spin wavefunctions. We write

$$
\begin{aligned}
e^{i \boldsymbol{\kappa} \cdot \mathbf{R}_{j}} & =e^{i \boldsymbol{\kappa} \cdot\left(\mathbf{R}_{j}^{(0)}+\mathbf{u}_{j}\right)} \approx e^{i \boldsymbol{\kappa} \cdot \mathbf{R}_{j}^{(0)}} e^{-\frac{1}{2}\left(\boldsymbol{\kappa} \cdot u_{j}\right)^{2}}\left[1+i\left(\boldsymbol{\kappa} \cdot \mathbf{u}_{j}\right)\right] \\
& \approx e^{-W} e^{i \boldsymbol{\kappa} \cdot \mathbf{R}_{j}^{(0)}}\left[1+i\left(\boldsymbol{\kappa} \cdot \mathbf{u}_{j}\right)\right]
\end{aligned}
$$

where $\mathbf{R}_{j}^{(0)}$ is the equilibrium value of $\mathbf{R}_{j}$ and $W \approx \frac{1}{6} \kappa^{2}\left\langle u^{2}\right\rangle \equiv \frac{1}{6} \kappa^{2}\left\langle u_{x}^{2}+u_{y}^{2}+u_{z}^{2}\right\rangle$. Since spherical harmonics of degree higher than two do not affect the manifolds of $J=0$ or $J=1$, we now use

$$
\cos \left(\frac{1}{2} \boldsymbol{\kappa} \cdot \boldsymbol{\rho}\right)=j_{0}\left(\frac{1}{2} \kappa \rho\right)-4 \pi j_{2}\left(\frac{1}{2} \kappa \rho\right) \sum_{\nu} Y_{2}^{n}(\hat{\boldsymbol{\kappa}})^{*} Y_{2}^{n}(\hat{\boldsymbol{\rho}})
$$

and

$$
\sin \left(\frac{1}{2} \boldsymbol{\kappa} \cdot \boldsymbol{\rho}\right)=4 \pi j_{1}\left(\frac{1}{2} \kappa \rho\right) \sum_{\nu} Y_{1}^{n}(\hat{\boldsymbol{\kappa}})^{*} Y_{1}^{n}(\hat{\boldsymbol{\rho}})
$$

We expand in displacements to get

$$
\begin{aligned}
\mathcal{S}_{0 \rightarrow 1, j}= & (4 \pi)^{2} A \sum_{J_{i}=0, J_{f}=1} P_{i} \delta\left(E-E_{i}+E_{f}\right)\left\langle f\left|\left[1+i \boldsymbol{\kappa} \cdot \mathbf{u}_{j}\right] \sum_{\nu} Y_{1}^{\nu}(\hat{\boldsymbol{\kappa}})^{*} Y_{1}^{\nu}(\hat{\boldsymbol{\rho}})\right| i\right\rangle \\
\times & \left\langle i\left|\left[1-i \boldsymbol{\kappa} \cdot \mathbf{u}_{j}\right] \sum_{\mu} Y_{1}^{\mu}(\hat{\boldsymbol{\kappa}}) Y_{1}^{\mu}(\hat{\boldsymbol{\rho}})^{*}\right| f\right\rangle \\
\mathcal{S}_{1 \rightarrow 0, j}= & (4 \pi)^{2} B \sum_{J_{i}=1, J_{f}=0} P_{i} \delta\left(E-E_{i}+E_{f}\right)\langle f|\left[1+i \boldsymbol{\kappa} \cdot \mathbf{u}_{j}\right] \\
& \sum_{\nu} Y_{1}^{\nu}(\hat{\boldsymbol{\kappa}})^{*} Y_{1}^{\nu}(\hat{\boldsymbol{\rho}})|i\rangle\left\langle i\left|\left[1-i \boldsymbol{\kappa} \cdot \mathbf{u}_{j}\right] \sum_{\mu} Y_{1}^{\mu}(\hat{\boldsymbol{\kappa}}) Y_{1}^{\mu}(\hat{\boldsymbol{\rho}})^{*}\right| f\right\rangle
\end{aligned}
$$

where $A=\frac{3}{4} e^{-2 W}\left[b^{\prime} j_{1}\left(\frac{1}{2} \kappa \rho\right)\right]^{2}$ and $B=\frac{1}{4} e^{-2 W}\left[b^{\prime} j_{1}\left(\frac{1}{2} \kappa \rho\right)\right]^{2}$ and 


$$
\begin{aligned}
\mathcal{S}_{1 \rightarrow 1, j}= & \frac{1}{2}\left(b^{\prime}\right)^{2} \sum_{J_{i}=1, J_{f}=1} P_{i} \delta\left(E-E_{i}+E_{f}\right) \\
& \times\left\langle f\left|\left[1+i \boldsymbol{\kappa} \cdot \mathbf{u}_{j}\right]\left[j_{0}\left(\frac{1}{2} \kappa \rho\right)+4 \pi j_{2}\left(\frac{1}{2} \kappa \rho\right) \sum_{\nu} Y_{2}^{\nu}(\hat{\boldsymbol{\kappa}})^{*} Y_{2}^{\nu}(\hat{\boldsymbol{\rho}})\right]\right| i\right\rangle \\
& \times\left\langle i\left|\left[1-i \boldsymbol{\kappa} \cdot \mathbf{u}_{j}\right]\left[j_{0}\left(\frac{1}{2} \kappa \rho\right)+4 \pi j_{2}\left(\frac{1}{2} \kappa \rho\right) \sum_{\mu} Y_{2}^{\mu}(\hat{\boldsymbol{\kappa}}) Y_{2}^{\mu}(\hat{\boldsymbol{\rho}})^{*}\right]\right| f\right\rangle,
\end{aligned}
$$

Since the phonon energy is much larger than the orientational energy, we may classify contributions according the number of phonons that are involved. In the notation of Eq. (C6) we write

$$
\mathcal{S}_{\beta}=\mathcal{S}_{\beta}^{(0)}+\mathcal{S}_{\beta}^{(1)}
$$

where $\mathcal{S}_{\beta}^{(0)}$ corresponds to a zero-phonon process and $\mathcal{S}_{\beta}^{(1)}$ to a process in which one phonon is created or destroyed. Thus

$$
\begin{aligned}
& \mathcal{S}_{0 \rightarrow 1}^{(0)}=(4 \pi)^{2} A \sum_{J_{i}=0, J_{f}=1} P_{i} \delta\left(E-E_{i}+E_{f}\right) \sum_{\mu \nu}\left\langle f\left|Y_{1}^{\nu}(\hat{\boldsymbol{\rho}})\right| i\right\rangle\left\langle i\left|Y_{1}^{\mu}(\hat{\boldsymbol{\rho}})^{*}\right| f\right\rangle Y_{1}^{\nu}(\hat{\boldsymbol{\kappa}})^{*} Y_{1}^{\mu}(\hat{\boldsymbol{\kappa}}) \\
& \mathcal{S}_{0 \rightarrow 1}^{(1)}=(4 \pi)^{2} A \sum_{J_{i}=0, J_{f}=1} P_{i} \delta\left(E-E_{i}+E_{f}\right) \\
& \\
& \quad \times \sum_{\mu \nu \alpha \beta}\left\langle f\left|u_{\alpha}^{*} Y_{1}^{\nu}(\hat{\boldsymbol{\rho}})\right| i\right\rangle\left\langle i\left|u_{\beta}^{*} Y_{1}^{\mu}(\hat{\boldsymbol{\rho}})^{*}\right| f\right\rangle \kappa_{\alpha} \kappa_{\beta} Y_{1}^{\nu}(\hat{\boldsymbol{\kappa}})^{*} Y_{1}^{\mu}(\hat{\boldsymbol{\kappa}}) .
\end{aligned}
$$

Here and below we use spherical components of a vector $\mathbf{v}: v_{ \pm 1}=\mp\left(v_{x} \pm i v_{y}\right) / \sqrt{2}$ and $v_{0}=v_{z}$. Similarly

$$
\begin{gathered}
\mathcal{S}_{1 \rightarrow 0}^{(0)}=(4 \pi)^{2} B \sum_{J_{i}=1, J_{f}=0} P_{i} \delta\left(E-E_{i}+E_{f}\right) \sum_{\mu \nu}\left\langle f\left|Y_{1}^{\nu}(\hat{\boldsymbol{\rho}})\right| i\right\rangle\left\langle i\left|Y_{1}^{\mu}(\hat{\boldsymbol{\rho}})^{*}\right| f\right\rangle Y_{1}^{\nu}(\hat{\boldsymbol{\kappa}})^{*} Y_{1}^{\mu}(\hat{\boldsymbol{\kappa}}) \\
\mathcal{S}_{1 \rightarrow 0}^{(1)}=(4 \pi)^{2} B \sum_{J_{i}=1, J_{f}=0} P_{i} \delta\left(E-E_{i}+E_{f}\right) \\
\quad \times \sum_{\mu \nu}\left\langle f\left|u_{\alpha}^{*} Y_{1}^{\nu}(\hat{\boldsymbol{\rho}})\right| i\right\rangle\left\langle i\left|u_{\beta}^{*} Y_{1}^{\mu}(\hat{\boldsymbol{\rho}})^{*}\right| f\right\rangle \kappa_{\alpha} \kappa_{\beta} Y_{1}^{\nu}(\hat{\boldsymbol{\kappa}})^{*} Y_{1}^{\mu}(\hat{\boldsymbol{\kappa}}), \\
S_{1 \rightarrow 1}^{(0)}=(4 \pi)^{2} C \sum_{J_{i}=1, J_{f}=1} P_{i} \delta\left(E-E_{i}+E_{f}\right) \sum_{\mu, \nu}\left\langle f\left|Y_{2}^{\mu}(\hat{\boldsymbol{\rho}})\right| i\right\rangle\left\langle i\left|Y_{2}^{\nu}(\hat{\boldsymbol{\rho}})\right| f\right\rangle Y_{2}^{\mu}(\hat{\boldsymbol{\kappa}})^{*} Y_{2}^{\nu}(\hat{\boldsymbol{\kappa}})^{*},
\end{gathered}
$$

where $C=\frac{1}{2} e^{-2 W}\left[b^{\prime} j_{2}\left(\frac{1}{2} \kappa \rho\right)\right]^{2}$ and 


$$
\begin{aligned}
& \mathcal{S}_{1 \rightarrow 1}^{(1)}=D_{0} \sum_{J_{i}=1, J_{f}=1} P_{i} \delta\left(E-E_{i}+E_{f}\right) \sum_{\alpha \beta}\left\langle f\left|u_{\alpha}^{*}\right| i\right\rangle\left\langle i\left|u_{\beta}\right| f\right\rangle \kappa_{\alpha}^{*} \kappa_{\beta} \\
& +4 \pi D_{1} \sum_{J_{i}=1, J_{f}=1} P_{i} \delta\left(E-E_{i}+E_{f}\right) \sum_{\mu \alpha \beta}\left\langle f\left|u_{\alpha}^{*} Y_{2}^{\mu}(\hat{\boldsymbol{\rho}})\right| i\right\rangle\left\langle i\left|u_{\beta}^{*}\right| f\right\rangle \kappa_{\alpha} \kappa_{\beta} Y_{2}^{\mu}(\hat{\boldsymbol{\kappa}})^{*} \\
& +4 \pi D_{1} \sum_{J_{i}=1, J_{f}=1} P_{i} \delta\left(E-E_{i}+E_{f}\right) \sum_{\mu \alpha \beta}\left\langle f\left|u_{\alpha}^{*}\right| i\right\rangle\left\langle i\left|u_{\beta}^{*} Y_{2}^{\mu}(\hat{\boldsymbol{\rho}})\right| f\right\rangle \kappa_{\alpha} \kappa_{\beta} Y_{2}^{\mu}(\hat{\boldsymbol{\kappa}})^{*} \\
& \quad+(4 \pi)^{2} D_{2} \sum_{J_{i}=1, J_{f}=1} P_{i} \delta\left(E-E_{i}+E_{f}\right) \sum_{\mu \nu \alpha \beta}\left\langle f\left|u_{\alpha}^{*} Y_{2}^{\nu}(\hat{\boldsymbol{\rho}})^{*}\right| i\right\rangle\left\langle i\left|u_{\beta}^{*} Y_{2}^{\mu}(\hat{\boldsymbol{\rho}})\right| f\right\rangle \kappa_{\alpha} \kappa_{\beta} Y_{2}^{\mu}(\hat{\boldsymbol{\kappa}})^{*} Y_{2}^{\nu}(\hat{\boldsymbol{\kappa}}) \\
& =\sum_{n=1}^{4} S_{1 \rightarrow 1 ; n}^{(1)},
\end{aligned}
$$

where $D_{n}=\frac{1}{2}\left(b^{\prime}\right)^{2} e^{-2 W} j_{0}\left(\frac{1}{2} \kappa \rho\right)^{2-n} j_{2}\left(\frac{1}{2} \kappa \rho\right)^{n}$ and $S_{1 \rightarrow 1 ; n}^{(1)}$ is the contribution to the one-phonon ortho cross section from the $n$th term in the first equality.

Note the existence of terms in which a phonon and a roton are created, the system evolves and finally a phonon is destroyed. This type of process can only occur when the system

supports roton-phonon interactions. All the terms $\mathcal{S}_{1 \rightarrow 1 ; n}^{(1)}$ correspond approximately to the phonon energy.

\section{POWDER AVERAGE AT LOW TEMPERATURE}

Here we restrict attention to the energy loss spectrum at low temperature when there are no thermal phonons present. Also, we now take the powder average. This corresponds to actual experimental situation in Ref. 5 , but would also be a reasonable approximation to take account of the differently oriented symmetry axes of the various octahedral interstitial sites. Below we calculate the cross sections for the following processes; (a) energy loss by conversion, (b) energy gain by conversion, (c) single phonon energy loss, and finally (d) zero phonon transition from $(J=1, M=0)$ to $(J=1, M= \pm 1)$.

\section{a. Energy Loss by Conversion}

We have

$$
\left\langle\mathcal{S}_{0 \rightarrow 1}^{(0)}\right\rangle=(4 \pi) A \sum_{J_{i}=0, J_{f}=1} P_{i} \delta\left(E-E_{i}+E_{f}\right) \sum_{\mu}\left\langle f\left|Y_{1}^{\mu}(\hat{\boldsymbol{\rho}})\right| i\right\rangle\left\langle i\left|Y_{1}^{\mu}(\hat{\boldsymbol{\rho}})^{*}\right| f\right\rangle
$$


where \langle\rangle indicates a powder average. The initial state is the $J=0, J_{z}=0$ zero-phonon state, which we write as

$$
\psi_{i}=\sum_{\mathbf{r}} c_{i}(\mathbf{r})\left|\mathbf{r} ; J=0 ; J_{z}=0\right\rangle,
$$

where $c_{i}(\mathbf{r})$ is the amplitude of the wave function at the mesh point $\mathbf{r}$. The final state is a $J=1$ zero-phonon state, which we similarly write as

$$
\psi_{f, m}=\sum_{\mathbf{r}, M} c_{f, m}(\mathbf{r}, M)\left|\mathbf{r} ; J=1 ; J_{z}=M\right\rangle
$$

and whose energy relative to the $J=0$ state is

$$
E_{f, m}=E_{c}+E_{m}
$$

If $E_{L}=-E$ is the energy loss, we may write

$$
\left\langle\mathcal{S}_{0 \rightarrow 1}^{(0)}\left(E_{L}\right)\right\rangle=A \sum_{m} \delta\left[E_{L}-\left(E_{c}+E_{m}\right)\right] \sum_{\mu}\left|\sum_{\mathbf{r}} c_{f, m}(\mathbf{r}, \mu)^{*} c_{i}(\mathbf{r})\right|^{2} .
$$

To a good approximation the zero-phonon wave functions for $J=1$ can be chosen to be composed of a single value of $J_{z}$. Thus we may label the wave functions so that $\mu=m$ :

$$
\left\langle\mathcal{S}_{0 \rightarrow 1}^{(0)}\left(E_{L}\right)\right\rangle=A \sum_{m} \delta\left[E_{L}-\left(E_{c}+E_{m}\right)\right]\left|\sum_{\mathbf{r}} c_{f, m}(\mathbf{r}, m)^{*} c_{i}(\mathbf{r})\right|^{2} .
$$

The corresponding integrated intensity is

$$
I^{(0)} \equiv \int d E_{L}\left\langle\mathcal{S}_{0 \rightarrow 1}^{(0)}\right\rangle=A \sum_{m}\left|\sum_{\mathbf{r}} c_{f, m}(\mathbf{r}, m)^{*} c_{i}(\mathbf{r})\right|^{2} .
$$

The inner product of the $J=0$ wave function and the spatial part of the $J=1$ zero-phonon states is essentially unity. So

$$
I^{(0)}=3 A
$$

\section{b. Energy Gain by Conversion}

Here we give a similar analysis of the energy-gain spectrum at low temperature due to ortho-para conversion. The derivation is similar to that for para-ortho conversion so we only quote the results: 


$$
\left\langle\mathcal{S}_{1 \rightarrow 0}^{(0)}\left(E_{L}\right)\right\rangle=B \sum_{m} P_{m} \delta\left[E-\left(E_{c}-E_{m}\right)\right]\left|\sum_{\mathbf{r}} c_{i, m}(\mathbf{r}, m)^{*} c_{f}(\mathbf{r})\right|^{2},
$$

where $P_{m}$ is the probability that the $J=1, J_{z}=m$ state is occupied and the role of initial and final states is interchanged from the para to ortho processes. The corresponding integrated intensity is

$$
\begin{aligned}
I^{(0)} & \equiv \int d E\left\langle\mathcal{S}_{1 \rightarrow 0}^{(0)}\right\rangle=B \sum_{m} P_{m}\left|\sum_{\mathbf{r}} c_{i, m}(\mathbf{r}, m)^{*} c_{f}(\mathbf{r})\right|^{2} \\
& =B .
\end{aligned}
$$

Since $B=3 A$ we see that ratio of the total cross section for ortho to para conversion to that of para to ortho conversion is $(1-x)$ to $x$, where $x$ is the ortho concentration. Normally the ratio of energy gain to energy loss cross sections follows the Boltzmann factor. Here, the populations are set by $x$ rather than by the temperature.

\section{c. Single Phonon Energy Loss}

We have the powder average of $S_{1 \rightarrow 1 ; 1}^{(1)}$ of Eq. (C21) as

$$
\left\langle S_{1 \rightarrow 1 ; 1}^{(1)}\right\rangle=\frac{1}{3} \kappa^{2} D_{0} \sum_{J_{i}=1, J_{f}=1} P_{i} \delta\left(E-E_{i}+E_{f}\right) \sum_{L}\left|\left\langle f\left|u_{L}\right| i\right\rangle\right|^{2}
$$

and the corresponding integrated intensity is

$$
I_{1}^{(1)} \equiv \int d E_{L}\left\langle S_{1 \rightarrow 1 ; 1}^{(1)}\left(E_{L}\right)\right\rangle=\frac{1}{3} \kappa^{2}\left\langle u^{2}\right\rangle D_{0}
$$

In terms of the amplitudes of the wave function on the mesh points, the above result is

$$
\left\langle S_{1 \rightarrow 1 ; 1}^{(1)}\right\rangle=\frac{1}{3} \kappa^{2} D_{0} \sum_{i, f} P_{i} \delta\left(E_{L}-E_{f}+E_{i}\right) \sum_{L}\left|\sum_{M, \mathbf{r}} c_{i}(M, \mathbf{r}) c_{f}(M, \mathbf{r}) r_{L}\right|^{2}
$$

Also we obtain the powder average of $S_{1 \rightarrow 1 ; 2}^{(1)}$ and $S_{1 \rightarrow 1 ; 3}^{(1)}$ of Eq. (C21) as

$$
\begin{aligned}
\left\langle S_{1 \rightarrow 1 ; 2}^{(1)}\left(E_{L}\right)\right\rangle= & \left\langle S_{1 \rightarrow 1 ; 3}^{(1)}\left(E_{L}\right)\right\rangle^{*}=-\frac{2}{5} \sqrt{\frac{8 \pi}{15}} D_{1} \kappa^{2} \sum_{i, f} P_{i} \delta\left(E_{L}-E_{f}+E_{i}\right) \\
& \times \sum_{M, M^{\prime}} C\left(112 ; M, M^{\prime}\right)(-1)^{M+M^{\prime}}\left\langle f\left|u_{-M} T_{2}^{M+M^{\prime}}(\mathbf{J})\right| i\right\rangle\left\langle i\left|u_{-M^{\prime}}\right| f\right\rangle,
\end{aligned}
$$

where the $T_{2}^{M}(\mathbf{J})$ are the operator equivalents of the spherical harmonics: 


$$
T_{2}^{ \pm 2}(\mathbf{J})=\sqrt{\frac{15}{32 \pi}} J_{ \pm}^{2}, \quad T_{2}^{ \pm 1}(\mathbf{J})=\mp \sqrt{\frac{15}{32 \pi}}\left(J_{z} J_{ \pm}+J_{ \pm} J_{z}\right), \quad T_{2}^{0}(\mathbf{J})=\sqrt{\frac{5}{16 \pi}}\left(3 J_{z}^{2}-2\right) .
$$

Here

$$
\left\langle i\left|u_{-M^{\prime}}\right| f\right\rangle=\sum_{\mu, \mathbf{r}} c_{i}(\mu, \mathbf{r})^{*} c_{f}(\mu, \mathbf{r}) r_{-M^{\prime}}
$$

and

$$
\left\langle f\left|u_{-M} T_{2}^{M+M^{\prime}}(\mathbf{J})\right| i\right\rangle=\frac{5}{\sqrt{8 \pi}} \sum_{\mu, \mathbf{r}} c_{i}(\mu, \mathbf{r}) c_{f}\left(\mu+M+M^{\prime}, \mathbf{r}\right)^{*} r_{-M} C\left(121 ; \mu, M+M^{\prime}\right),
$$

where we used

$$
\left\langle J=1 ; J_{z}=M\left|T_{2}^{L}(\boldsymbol{\rho})\right| J=1 ; J_{z}=M^{\prime}\right\rangle=\frac{5}{\sqrt{8 \pi}} \delta_{M, L+M^{\prime}} C\left(121 ; M^{\prime}, L\right) .
$$

We have the contributions to the integrated intensity

$$
\begin{aligned}
I_{2}^{(1)} & =I_{3}^{(1)^{*}}=\int d E_{L}\left\langle S_{1 \rightarrow 1 ; 2}^{(1)}\left(E_{L}\right)\right\rangle \\
& =-\frac{2}{5} \sqrt{\frac{8 \pi}{15}} D_{1} \kappa^{2} \sum_{i f} P_{i} \sum_{M, M^{\prime}} C\left(112 ; M, M^{\prime}\right)(-1)^{M+M^{\prime}}\left\langle i\left|u_{-M^{\prime}}\right| f\right\rangle\left\langle f\left|u_{-M} T_{2}^{M+M^{\prime}}(\mathbf{J})\right| i\right\rangle .
\end{aligned}
$$

Here the sum over final states should be restricted to single phonon states. Higher energy states make only a small contribution to this sum. So we make the closure approximation that the sum over $|f\rangle$ extends over all states, in which case

$$
I_{2}^{(1)}=-\frac{2}{5} \sqrt{\frac{8 \pi}{15}} D_{1} \kappa^{2} \sum_{i} P_{i} \sum_{M, M^{\prime}} C\left(112 ; M, M^{\prime}\right)(-1)^{M}\left\langle i\left|u_{M^{\prime}}^{*} u_{-M} T_{2}^{M+M^{\prime}}(\mathbf{J})\right| i\right\rangle .
$$

As illustrated by Eq. (20), the initial state $|i\rangle$ is dominantly comprised of a single value of $J_{z}$. Thus in Eq. C41 $M+M^{\prime}=0$ dominates. In addition, the system is nearly isotropic. Then $\sum_{M} C(112 ; M,-M)(-1)^{M}\left|u_{-M}\right|^{2}=0$. So, to a good approximation,

$$
I_{2}^{(1)}+I_{3}^{(1)}=0
$$

We have made several approximations, but our result for the total integrated intensity will not be much affected by these approximations.

Similarly, we get 


$$
\begin{aligned}
& \left\langle S_{1 \rightarrow 1 ; 4}^{(1)}\left(E_{L}\right)\right\rangle=\frac{16 \pi}{75} \kappa^{2} D_{2} \sum_{i, f, \mu, M} P_{i} \delta\left(E_{L}-E_{f}+E_{i}\right)\left|\left\langle f\left|u_{M} T_{2}^{\mu}(\mathbf{J})\right| i\right\rangle\right|^{2} \\
& -\frac{32 \pi}{25 \sqrt{21}} D_{2} \kappa^{2} \sum_{i, f, \nu, M, M^{\prime}} P_{i} \delta\left(E_{L}-E_{f}+E_{i}\right) C\left(112 ; M, M^{\prime}\right) C\left(222 ; M+M^{\prime}, \nu\right)(-1)^{M^{\prime}} \\
& \quad \times\left\langle f\left|u_{-M} T_{2}^{-\nu}(\mathbf{J})\right| i\right\rangle\left\langle f\left|u_{M^{\prime}} T_{2}^{-M-M^{\prime}-\nu}(\mathbf{J})\right| i\right\rangle^{*}
\end{aligned}
$$

We now evaluate the integrated intensity

$$
I_{4}^{(1)} \equiv \int d E_{L}\left\langle S_{1 \rightarrow 1 ; 4}^{(1)}\left(E_{L}\right)\right\rangle \equiv t_{1}+t_{2}
$$

where

$$
t_{1}=\frac{16 \pi}{75} \kappa^{2} D_{2} \sum_{i, f, \mu, M} P_{i}\left\langle i\left|u_{-M} T_{2}^{-\mu}(\mathbf{J})\right| f\right\rangle\left\langle f\left|u_{M} T_{2}^{\mu}(\mathbf{J})\right| i\right\rangle(-1)^{M+\mu}
$$

Making the closure approximation this is

$$
\begin{aligned}
t_{1} & =\frac{16 \pi}{75} \kappa^{2} D_{2} \sum_{i, \mu, M} P_{i}\left\langle i\left|u_{-M} u_{M} T_{2}^{-\mu}(\mathbf{J}) T_{2}^{\mu}(\mathbf{J})\right| i\right\rangle(-1)^{\mu+M} \\
& =\frac{2}{3} \kappa^{2} D_{2} \sum_{i} P_{i}\left\langle i\left|u_{-M} u_{M}\right| i\right\rangle(-1)^{M}=\frac{2}{3} \kappa^{2} D_{2}\left\langle u^{2}\right\rangle
\end{aligned}
$$

Similarly

$$
\begin{aligned}
t_{2}= & -\frac{32 \pi}{25 \sqrt{21}} D_{2} \kappa^{2} \sum_{i, M, M^{\prime} \nu} P_{i} C\left(112 ; M, M^{\prime}\right) C\left(222 ; M+M^{\prime}, \nu\right) \\
& \times(-1)^{M+\nu}\left\langle i\left|u_{M}^{*} T_{2}^{M+M^{\prime}+\nu}(\mathbf{J}) u_{-M} T_{2}^{-\nu}(\mathbf{J})\right| i\right\rangle
\end{aligned}
$$

Again, we treat $|i\rangle$ as having a single value of $J_{z}$, so that $M+M^{\prime}=0$. Also we again assume spatial isotropy, so that $\sum_{M} C(112 ; M,-M)(-1)^{M}\left|u_{-M}\right|^{2}=0$. Then $t_{2}=0$.

\section{d. Zero Phonon Ortho Cross Section}

Finally we consider the zero phonon contribution to the ortho to ortho cross section. Taking the powder average, we have

$$
\left\langle S_{1 \rightarrow 1}^{(0)}\right\rangle=4 \pi C \sum_{J_{i}=1} \sum_{J_{f}=1} P_{i} \delta\left(E-E_{i}+E_{f}\right) \sum_{\mu}\left\langle f\left|Y_{2}^{\mu}(\hat{\boldsymbol{\rho}})\right| i\right\rangle\left\langle i\left|Y_{2}^{-\mu}(\hat{\boldsymbol{\rho}})\right| f\right\rangle(-1)^{\mu}
$$

If $\delta$ is the energy of the $J_{z}= \pm 1$ levels relative to the $J_{z}=0$ level and if the Boltzmann probability of the state $\mid m>$ is $P_{m}$, then we may write 


$$
\begin{aligned}
\left\langle S_{1 \rightarrow 1}^{(0)}\right\rangle= & 4 \pi C P_{0} \delta(E+\delta) \sum_{m= \pm 1}\left[\left|\left\langle m\left|Y_{2}^{1}(\hat{\boldsymbol{\rho}})\right| 0\right\rangle\right|^{2}+\left|\left\langle m\left|Y_{2}^{-1}(\hat{\boldsymbol{\rho}})\right| 0\right\rangle\right|^{2}\right] \\
& +4 \pi C \delta(E-\delta) \sum_{m= \pm 1} P_{m}\left[\left|\left\langle m\left|Y_{2}^{1}(\hat{\boldsymbol{\rho}})\right| 0\right\rangle\right|^{2}+\left|\left\langle m\left|Y_{2}^{-1}(\hat{\boldsymbol{\rho}})\right| 0\right\rangle\right|^{2}\right],
\end{aligned}
$$

which gives

$$
\left\langle S_{1 \rightarrow 1}^{(0)}\right\rangle=\frac{6}{5} C P_{0} \delta(E+\delta)+\frac{6}{5} C P_{1} \delta(E-\delta)
$$

The ratio of cross sections at energy gain to those of energy loss does satisfy detailed balance because within the species $(J=1)$ we do maintain thermal equilibrium.

\section{INTENSITY RATIOS}

We develop an expression for the ratio, $r_{P}$, defined to be the integrated intensity due to phonon creation divided by that due to para-ortho conversion. Using the results for $I^{(n)}$ obtained above we have

$$
\begin{aligned}
r_{P} & =\frac{x \int d E_{L}\left\langle S_{1 \rightarrow 1}^{(1)}\left(E_{L}\right)\right\rangle}{(1-x) \int d E_{L}\left\langle S_{0 \rightarrow 1}^{(0)}\left(E_{L}\right)\right\rangle}=\frac{x\left[I_{1}^{(1)}+I_{4}^{(1)}\right]}{(1-x) I^{(0)}} \\
& =\frac{\frac{1}{3} \kappa^{2}\left\langle u^{2}\right\rangle D_{0}+\frac{2}{3} \kappa^{2}\left\langle u^{2}\right\rangle D_{2}}{3 A}\left(\frac{x}{1-x}\right) \\
& =\frac{2}{27} \kappa^{2}\left\langle u^{2}\right\rangle \frac{j_{0}\left(\frac{1}{2} \kappa \rho\right)^{2}+2 j_{2}\left(\frac{1}{2} \kappa \rho\right)^{2}}{j_{1}\left(\frac{1}{2} \kappa \rho\right)^{2}}\left(\frac{x}{1-x}\right) .
\end{aligned}
$$

The ratio $r_{C}$, defined to be the ortho to para conversion cross section in energy gain to that in energy loss due to para to ortho conversion, is given by

$$
r_{C}=\frac{x}{1-x} .
$$

Finally, we have $r_{J=1}$, defined to be the total cross section (counting both energy loss and energy gain) for transitions within the $J=1$ ground manifold divided by that due to para to ortho conversion, as

$$
r_{J=1}=\left(\frac{x}{1-x}\right) \frac{\frac{6}{5} C\left(P_{0}+P_{1}\right)}{3 A}=\left(P_{0}+P_{1}\right) r_{J=1}(T=0)
$$

where

$$
r_{J=1}(T=0)=\left(\frac{x}{1-x}\right) \frac{4 j_{2}\left(\frac{1}{2} \kappa \rho\right)^{2}}{15 j_{1}\left(\frac{1}{2} \kappa \rho\right)^{2}} .
$$




\section{REFERENCES}

${ }^{1}$ Q. Wang and J. K. Johnson, J. Chem. Phys. 110, 577 (1999).

${ }^{2}$ Q. Wang, S. R. Challa, D. S. Sholl, and J. K. Johnson, Phys. Rev. Lett. 82, 956 (1999).

${ }^{3}$ M. M. Calbi and M. W. Cole, Rev. Mod. Phys., 73, 857 (2001).

${ }^{4}$ A. D. Novaco and J. P. Wroblewski, Phys. Rev. B 39, 11364 (1989).

${ }^{5}$ S. A. FitzGerald, T. Yildirim, L. J. Santodonato, D. A. Neumann, J. R. D. Copley, J. J. Rush, and F. Trouw, Phys. Rev. B60, 6439 (1999).

${ }^{6}$ J. Van Kranendonk, Solid Hydrogen (Plenum, New York, 1983).

${ }^{7}$ R. M. Fleming, A. P. Ramirez, M. J. Rosseinsky, D. W. Murphy, R. C. Haddon, S. M. Zahurak, and A. V. Makhija, Nature 352, 787 (1991).

${ }^{8}$ P. A. Heiney, J. E. Fischer, A. R. McGhie, W. J. Romanow, A. M. Denenstein, J. P. McCauley, Jr., A. B. Smith III, and D. E. Cox, Phys. Rev. Lett. 66, 2911 (1991).

${ }^{9}$ R. Sachidanandam and A. B. Harris, Phys. Rev. Lett. 67, 1467 (1991).

${ }^{10}$ P. A. Heiney et al., Phys. Rev. Lett. 67, 1468 (1991).

${ }^{11}$ S. Liu, Y. J. Lu, M. M. Kappes, and J. A. Ibers, Science 254, 408 (1991).

12 W. I. F. David, R. M. Ibberson, J. C. Mathewman, K. Prassides, T. J. Dennis, J. P. Hare, H. W. Kroto, R. Taylor, and D. R. M. Walton, Nature (London) 353, 147 (1991).

${ }^{13}$ International Tables for Crystallography, edited by Theo Hahn (Riedel, Boston, 1983), Vol4.

${ }^{14}$ I. Holleman, G. von Heiden, E. H. T. Olthof, P. J. M. van Bentum, R. Engeln, G. H. Nachtegaal, A. P. Kentgens, B. H. Meier, A. van der Avoird, and G. Meijer, Phys. Rev. Lett. 79, 1138 (1997).

15 The Atom-Atom Potential Method, edited by A. J. Pertsin and A. I. Kitaigorodsky (SpringerVerlag, Berlin, 1986), p. 89.

${ }^{16}$ T. Yildirim and A. B. Harris, unpublished. 
${ }^{17}$ ARPACK is a collection of Fortran77 subroutines designed to solve large scale eigenvalue problems. The package is designed to compute a few eigenvalues and corresponding eigenvectors of a general $\mathrm{n}$ by $\mathrm{n}$ matrix A. More information about ARPACK and the code is available at http://www.caam.rice.edu/software/ARPACK

${ }^{18}$ C. Kittel Quantum Theory of Solids (Wiley, New York, 1963). See Chap. 7.

${ }^{19}$ M. Tinkham, Group Theory and Quantum Mechanics (McGraw-Hill, New York, 1964).

20 T. Nakamura, Progr. Theoret Phys. 14, 135 (1955).

${ }^{21}$ A. B. Harris and A. J. Berlinsky, Phys Rev. B16, 3791 (1977).

${ }^{22}$ D. N. Zubarev, Usp. Fiz. Nauk. 71, 71 (1957); [English Transl. Soviet Phys.-Usp. 3, 320 $(1960)]$.

${ }^{23}$ R. J. Elliott and W. M. Hartmann, Proc. Phys. Soc. (London) 90, 671 (1967).

${ }^{24}$ M. E. Rose, Elementary Theory of Angular Momentum. (Dover, 1995). 


\section{TABLES}

TABLE I. Effect of a spherical symmetric perturbation on $U_{3}$ states. The $U_{3}$ states are characterized by $N$, the total number of harmonic phonons. Wave functions in a spherical potential are characterized by angular momentum $K$. Here we give the effect of the perturbation $\Delta(r / \sigma)^{4}$, where $\left\langle r^{2}\right\rangle=3 \sigma^{2}$ for the isotropic harmonic oscillator in three spatial dimensions. In the last column we give the shift in the average energy of the multiplet of states of a given value of $N$.

\begin{tabular}{|lccr|}
\hline \hline$N$ & $N, K$ & Energy & Avg $E$ \\
\hline \hline 0 & $(0,0)$ & $15 \Delta$ & $15 \Delta$ \\
\hline \hline 1 & $(1,1)$ & $35 \Delta$ & $35 \Delta$ \\
\hline \hline 2 & $(2,0)$ & $75 \Delta$ & $65 \Delta$ \\
& $(2,2)$ & $63 \Delta$ & $105 \Delta$ \\
\hline \hline 3 & $(3,1)$ & $119 \Delta$ & \\
& $(3,3)$ & $99 \Delta$ & \\
\hline \hline
\end{tabular}


TABLE II. Energy level systematics for a $(J=0)$ molecule in an octahedral interstitial site of $\mathrm{C}_{60}$. Here we show the removal of degeneracy from a manifold of initial symmetry $\mathcal{I}$ to manifolds of final symmetry $\mathcal{F}$ due to a perturbation $V$, as calculated in lowest-order perturbation theory. Here $N$ is the total number of phonons, $K$ is the angular momentum, and the other group theoretical labels are as in Fig. 1. We give a typical eigenfunction $\psi$ to illustrate the symmetry. Here $d$ is the degeneracy (Deg) of the manifold and $\sigma^{2}=\left\langle x^{2}\right\rangle=\left\langle y^{2}\right\rangle=\left\langle z^{2}\right\rangle$. Here the coordinate axes coincide with the cubic [100] directions.

\begin{tabular}{|c|c|c|c|c|c|c|}
\hline $\mathcal{I}$ & $\psi$ & $V$ & $\psi$ & $\mathcal{F}$ & Deg & Energy \\
\hline$N=2, K=2$ & $r^{2} Y_{2}^{M}(\Omega)$ & $\kappa\left(x^{4}+y^{4}+z^{4}-\frac{3}{5} r^{4}\right)$ & $\begin{array}{c}\left(x^{2}-y^{2}\right) \\
x y\end{array}$ & $\begin{array}{l}\mathrm{E}_{\mathrm{g}} \\
\mathrm{T}_{2 \mathrm{~g}}\end{array}$ & $\begin{array}{l}2 \\
3\end{array}$ & $\begin{array}{c}\frac{36}{5} \kappa \sigma^{4} \\
-\frac{24}{5} \kappa \sigma^{4}\end{array}$ \\
\hline$N=3, K=3$ & $r^{3} Y_{3}^{M}(\Omega)$ & $\kappa\left(x^{4}+y^{4}+z^{4}-\frac{3}{5} r^{4}\right)$ & $\begin{array}{c}\left(x^{3}-\frac{3}{5} x r^{2}\right) \\
x\left(y^{2}-z^{2}\right) \\
x y z\end{array}$ & $\begin{array}{l}\mathrm{T}_{1 \mathrm{u}} \\
\mathrm{T}_{2 \mathrm{u}} \\
\mathrm{A}_{\mathrm{u}}\end{array}$ & $\begin{array}{l}3 \\
3 \\
1\end{array}$ & $\begin{array}{l}\frac{36}{5} \kappa \sigma^{4} \\
-\frac{12}{5} \kappa \sigma^{4} \\
-\frac{72}{5} \kappa \sigma^{4} \\
\end{array}$ \\
\hline$N=1, K=1, \mathrm{~T}_{1 \mathrm{u}}$ & $r Y_{1}^{M}(\Omega)$ & $\lambda(x y+y z+z x)$ & & $\begin{array}{l}\mathrm{E}_{\mathrm{u}} \\
\mathrm{A}_{\mathrm{u}}\end{array}$ & $\begin{array}{l}2 \\
1\end{array}$ & $\begin{array}{l}-\lambda \sigma^{2} \\
2 \lambda \sigma^{2}\end{array}$ \\
\hline$N=2, K=2, \mathrm{~T}_{2 \mathrm{~g}}$ & $x y$ & $\lambda(x y+y z+z x)$ & & $\begin{array}{l}\mathrm{E}_{\mathrm{g}} \\
\mathrm{A}_{\mathrm{g}} \\
\end{array}$ & $\begin{array}{l}2 \\
1 \\
\end{array}$ & $\begin{array}{l}-\lambda \sigma^{2} \\
2 \lambda \sigma^{2} \\
\end{array}$ \\
\hline$N=3, K=3, \mathrm{~T}_{2 \mathrm{u}}$ & $x\left(y^{2}-z^{2}\right)$ & $\lambda(x y+y z+z x)$ & & $\begin{array}{l}\mathrm{E}_{\mathrm{u}} \\
A_{\mathrm{u}}\end{array}$ & $\begin{array}{l}2 \\
1\end{array}$ & $\begin{array}{l}\frac{3}{2} \lambda \sigma^{2} \\
-3 \lambda \sigma^{2}\end{array}$ \\
\hline$N=3, K=3, \mathrm{~T}_{1 \mathrm{u}}$ & $\left(x^{3}-\frac{3}{5} x r^{2}\right)$ & $\lambda(x y+y z+z x)$ & & $\begin{array}{l}\mathrm{E}_{\mathrm{u}} \\
\mathrm{A}_{\mathrm{u}}\end{array}$ & $\begin{array}{l}2 \\
1\end{array}$ & $\begin{array}{l}\frac{3}{10} \lambda \sigma^{2} \\
-\frac{3}{5} \lambda \sigma^{2}\end{array}$ \\
\hline$N=3, K=1, \mathrm{~T}_{1 \mathrm{u}}$ & $x\left(r^{2}-\frac{1}{3} \sigma^{2}\right)$ & $\lambda(x y+y z+z x)$ & & $\begin{array}{l}\mathrm{E}_{\mathrm{u}} \\
\mathrm{A}_{\mathrm{u}}\end{array}$ & $\begin{array}{l}2 \\
1\end{array}$ & $\begin{array}{c}3 \lambda \sigma^{2} \\
-6 \lambda \sigma^{2}\end{array}$ \\
\hline
\end{tabular}


TABLE III. Phonon levels for a $(J=0) \mathrm{H}_{2}$ molecule in an octahedral site for orientationally ordered and disordered $\mathrm{C}_{20}$ respectively. Energies $(\mathrm{meV})$ are with respect to the ground state energy $\mathrm{E}_{0,1}$. The symmetry of each manifold of degenerate levels can be read from Fig. 1.

\begin{tabular}{lrrr}
\hline \hline & $\mathrm{E}_{N, \alpha}$ & Octahedral & $\mathrm{Pa} \overline{3}\left(\mathrm{~S}_{6}\right)$ \\
\hline $\mathrm{N}$ & $\alpha$ & & \\
\hline 1 & 1 & 14.38 & 13.16 \\
1 & 2,3 & 14.38 & 14.47 \\
\hline 2 & 1 & 28.26 & 26.69 \\
2 & 2,3 & 28.26 & 27.49 \\
2 & 4 & 30.69 & 30.41 \\
2 & 5,6 & 31.73 & 31.39 \\
\hline 3 & 1 & 41.62 & 40.10 \\
3 & 2,3 & 44.39 & $42.40^{\mathrm{a}}$ \\
3 & 4 & 44.39 & $42.42^{\mathrm{a}}$ \\
3 & 5,6 & 45.23 & 44.40 \\
3 & 7 & 45.23 & 45.83 \\
3 & 8,9 & 50.07 & 49.36 \\
3 & 10 & 50.07 & 49.85 \\
\hline \hline
\end{tabular}

a) These energies are accidentally almost identical. However, group theory indicates that these levels are generically nondegenerate. 
TABLE IV. Matrix elements of $<N \alpha\left|A_{2}^{\tau}(r)\right| 01>$ (in meV) for $\mathrm{H}_{2}$ in octahedral and $\mathrm{S}_{6}$ potential, respectively. Elements not listed are expected to be zero by symmetry. Numerically such elements were found to be very small. For this table the wave functions within each degenerate manifold were chosen to make the matrix elements of $A_{2}^{\tau}(\mathbf{r})$ as simple as possible. For Pa $\overline{3}$ symmetry, the z-axis is taken to be the local three-fold axis. Therefore the octahedral wavefunctions are not necessarily identical to the $\mathrm{Pa} \overline{3}$ wavefunctions.

\begin{tabular}{|c|c|c|c|c|}
\hline \multicolumn{3}{|c|}{$<N \alpha\left|A_{2}^{\tau}(\mathbf{r})\right| 01>$} & \multirow[t]{2}{*}{ Octahedral $^{\mathrm{a}}$} & \multirow[t]{2}{*}{$\operatorname{Pa} \overline{3}\left(\mathrm{~S}_{6}\right)$} \\
\hline $\mathrm{N}$ & $\alpha$ & $\tau$ & & \\
\hline 0 & 1 & 0 & $(0,0)$ & $(-1.286,0)$ \\
\hline 2 & 1 & 0 & $(0,0)$ & $(-0.506,0)$ \\
\hline 2 & 1 & 1 & $(\alpha, 0)$ & $(0,0)$ \\
\hline 2 & 1 & 2 & $(0,0)$ & $(0,0)$ \\
\hline 2 & 2 & 1 & $(0, \alpha)$ & $(-0.176,-0.106)$ \\
\hline 2 & 3 & 2 & $(0, \alpha)$ & $(2.413,-2.388)$ \\
\hline 2 & 4 & 0 & $(0,0)$ & $(-0.224,0)$ \\
\hline 2 & 5 & 0 & $(0,0)$ & $(0,0)$ \\
\hline 2 & 5 & 2 & $(\beta, 0)$ & $(-6.500,-1.754)$ \\
\hline 2 & 6 & 0 & $(\sqrt{2} \beta, 0)$ & $(0,0)$ \\
\hline 2 & 6 & 1 & $(0,0)$ & $(-8.862,-1.400)$ \\
\hline
\end{tabular}

a) Our numerical results give $\alpha=0.859$ and $\beta=5.662$ (in meV). 
TABLE V. Non-zero matrix elements of $<1 \alpha\left|A_{2}^{\tau}(r)\right| 1 \beta>$ (in meV) for $\mathrm{H}_{2}$ in octahedral and $\mathrm{S}_{6}$ potential, respectively. We also note that $<1 \alpha\left|A_{2}^{\tau}(r)\right| 1 \beta>=<1 \beta\left|A_{2}^{\tau}(r)\right| 1 \alpha>$ and $<1 \alpha\left|A_{2}^{-\tau}(r)\right| 1 \beta>=(-1)^{\tau}<1 \alpha\left|A_{2}^{\tau}(r)\right| 1 \beta>^{*}$. For the octahedral symmetry, the wave functions within each degenerate manifold were chosen to make the matrix elements of $A_{2}^{\tau}(\mathbf{r})$ as simple as possible.

\begin{tabular}{|c|c|c|c|c|}
\hline & $<1 \alpha\left|A_{2}^{\tau}(r)\right| 1 \beta>$ & & Octahedral & $\operatorname{Pa} \overline{3}\left(\mathrm{~S}_{6}\right)$ \\
\hline$\tau$ & $\alpha$ & $\beta$ & & \\
\hline 0 & 1 & 1 & $(-3.853,0)$ & $(-2.020,0)$ \\
\hline 0 & 2 & 2 & $(-3.853,0)$ & $(-1.326,0)$ \\
\hline 0 & 3 & 3 & $(7.708,0)$ & $(-1.326,0)$ \\
\hline 1 & 1 & 2 & $(0,0)$ & $(-2.109,-1.131)$ \\
\hline 1 & 1 & 3 & $(0,0.881)$ & $(1.137,-2.104)$ \\
\hline 1 & 2 & 2 & $(0,0)$ & $(1.534,2.440)$ \\
\hline 1 & 2 & 3 & $(-0.881,0)$ & $(2.435,-1.533)$ \\
\hline 1 & 3 & 3 & $(0,0)$ & $(-1.531,-2.442)$ \\
\hline 2 & 1 & 1 & $(-4.119,0)$ & $(0,0)$ \\
\hline 2 & 1 & 2 & $(0,0.880)$ & $(-0.225,-3.022)$ \\
\hline 2 & 1 & 3 & $(0,0)$ & $(-3.022,0.226)$ \\
\hline 2 & 2 & 2 & $(0,0)$ & $(0.560,0.835)$ \\
\hline 2 & 2 & 3 & $(0,0)$ & $(-0.830,0.556)$ \\
\hline 2 & 3 & 3 & $(0,0)$ & $(-0.565,-0.831)$ \\
\hline
\end{tabular}


TABLE VI. Shift of the center of gravity (CG) and splitting (in meV) of the $(J=1, N=0$ ) manifold when the nominally octahedral site has octahedral and $\mathrm{S}_{6}$ symmetry.

\begin{tabular}{|c|c|c|c|}
\hline \multirow[b]{2}{*}{ Quantity } & \multicolumn{2}{|c|}{ Our Calculations } & \multirow{2}{*}{$\begin{array}{c}\text { Experiment }^{\mathrm{d}} \\
\operatorname{Pa} \overline{3}\left(\mathrm{~S}_{6}\right) \\
\end{array}$} \\
\hline & Octahedral $\left(\mathrm{O}_{\mathrm{h}}\right)$ & $\mathrm{Pa} \overline{3}\left(\mathrm{~S}_{6}\right)$ & \\
\hline Shift of $C G^{a}$ & -0.134 & -0.141 & 0.35 \\
\hline Shift of $\mathrm{CG}^{\mathrm{b}}$ & & -0.16 & 0.35 \\
\hline Splitting 1st order ${ }^{\mathrm{c}}$ & 0 & 0.487 & \\
\hline 2nd order ${ }^{\mathrm{c}}$ & 0 & -0.010 & \\
\hline total $^{\mathrm{c}}$ & 0 & 0.477 & 0.70 \\
\hline total $^{\mathrm{b}}$ & 0 & 0.46 & 0.70 \\
\hline
\end{tabular}
a) Perturbation result of Eq. (16).
b) Obtained by direct diagonalization of Eq. (3)
c) Perturbation result of Eq. (17).
d) From Ref. 5 . 
TABLE VII. Energy (in meV) of $J=1$ single-phonon states where zero-of-energy is taken to be $2 \mathrm{~B}$.

\begin{tabular}{|c|cc|c|cc}
\hline \hline \multicolumn{3}{c}{ Octahedral $\left(\mathrm{O}_{\mathrm{h}}\right)$} & \multicolumn{3}{c}{${\text { Paj }\left(\mathrm{S}_{6}\right)}$} \\
\hline$\Psi$ & Full Mesh $^{\mathrm{a}}$ & Perturbation $^{\mathrm{b}}$ & $\Psi$ & Full Mesh $^{\mathrm{a}}$ & Perturbation $^{\mathrm{b}}$ \\
\hline$T_{\mathrm{gg}}$ & 13.11 & 13.14 & $\mathrm{~A}_{\mathrm{g}}$ & 12.59 & 12.52 \\
$T_{1 \mathrm{~g}}$ & 13.11 & 13.14 & $\mathrm{E}_{\mathrm{g}}$ & 12.82 & 12.87 \\
$T_{1 \mathrm{~g}}$ & 13.11 & 13.14 & $\mathrm{E}_{\mathrm{g}}$ & 12.82 & 12.87 \\
$T_{2 \mathrm{~g}}$ & 13.6 & 13.68 & $\mathrm{E}_{\mathrm{g}}$ & 13.47 & 13.51 \\
$T_{2 \mathrm{~g}}$ & 13.6 & 13.68 & $\mathrm{E}_{\mathrm{g}}$ & 13.47 & 13.51 \\
$T_{2 \mathrm{~g}}$ & 13.6 & 13.68 & $\mathrm{Ag}_{\mathrm{g}}$ & 14.20 & 14.16 \\
$A_{\mathrm{g}}$ & 15.61 & 15.78 & $\mathrm{~A}_{\mathrm{g}}$ & 15.75 & 15.31 \\
$E_{\mathrm{g}}$ & 16.46. & 16.60 & $\mathrm{E}_{\mathrm{g}}$ & 16.60 & 15.79 \\
$E_{\mathrm{g}}$ & 16.46. & $\mathrm{E}_{\mathrm{g}}$ & 16.60 & 15.79 \\
\hline \hline
\end{tabular}

a) Solution to Eq. (3) for the three component wavefunction on a mesh of points.

b) Solution to Eq. (5) using wavefunctions and energies for a $J=0$ molecule as previously determined numerically on a mesh of points. 
TABLE VIII. Basis functions within the one phonon $(J=1)$ manifold. Here $x, y$, and $z$ are the one phonon states with a single excitation in the phonon associated with the $x, y$, and $z$ direction, respectively. ${ }^{(a)}$ In terms of the $m_{J}$ states (denoted $\left.\left|m_{J}\right\rangle\right)$ within $(J=1)$ we have $X \equiv(|-1\rangle-|1\rangle) / \sqrt{2}$, $Y \equiv i(|1\rangle+|-1\rangle) / \sqrt{2}$, and $Z \equiv|0\rangle$.

\begin{tabular}{|c|c|}
\hline \multicolumn{2}{|r|}{$\mathrm{O}_{\mathrm{h}}$ Symmetry $^{\mathrm{a}}$} \\
\hline $\mathrm{T}_{2 \mathrm{~g}}$ & $(x Z+z X),(y Z+z Y),(x Y+y X)$ \\
\hline $\mathrm{T}_{1 \mathrm{~g}}$ & $(x Z-z X),(y Z-z Y),(x Y-y X)$ \\
\hline $\mathrm{E}_{\mathrm{g}}$ & $(2 z Z-y Y-x X),(x X-y Y)$ \\
\hline $\mathrm{A}_{\mathrm{g}}$ & $(x X+y Y+z Z)$ \\
\hline \multicolumn{2}{|r|}{ Pa⿳亠丷厂 Symmetry ${ }^{\mathrm{b}}$} \\
\hline $\mathrm{E}_{\mathrm{g}}$ and $\mathrm{E}_{\mathrm{g}}^{*}$ & $(x Z+z X, y Z+z Y),(x Z-z X, y Z-z Y),(x X-y Y, x Y+y X)$ \\
\hline$A_{g}$ & $z Z, x X+y Y, x Y-y X$ \\
\hline
\end{tabular}

a) The $x, y$, and $z$ directions are taken to coincide with the four-fold axis of $\mathrm{O}_{\mathrm{h}}$.

b) The $z$ direction coincides with the three-fold axis of $\mathrm{S}_{6}$. 


\section{FIGURES}

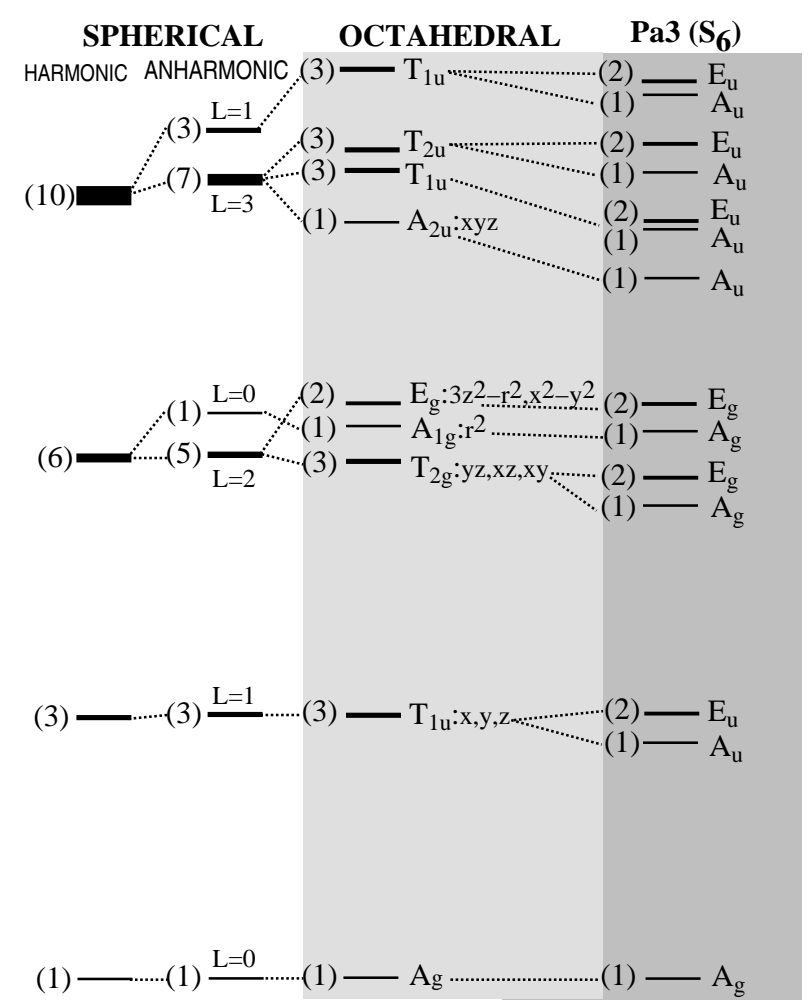

FIG. 1. Energy levels of a spherical $(J=0) \mathrm{H}_{2}$ molecule confined in various ways. Here $(g)$ is the degeneracy and the symmetry labels are given. Left: The molecule is in a spherical harmonic potential $V(\mathbf{r})=\frac{1}{2} k\left(x^{2}+y^{2}+z^{2}\right)$ or an anharmonic spherically symmetric potential (i. e. a generic spherically symmetric potential). For a harmonic spherically symmetric potential the energy depends only on $N$, the total number of phonon excitations in the oscillators along the three coordinate directions. For a spherically symmetric potential eigenstates are characterized by their total orbital angular momentum $L$. Center: the molecule is in a potential appropriate to the octahedral interstitial site of orientationally disordered $(\mathrm{Fm} 3 \mathrm{~m})$ solid $\mathrm{C}_{60}$. Right: the molecule is in a potential appropriate to the octahedral interstitial site of orientationally ordered $(\mathrm{Pa} \overline{3})$ solid $\mathrm{C}_{60}$, in which case the site symmetry is $\mathrm{S}_{6}$. The potentials used for the interstitial cases are discussed in Appendix A. 


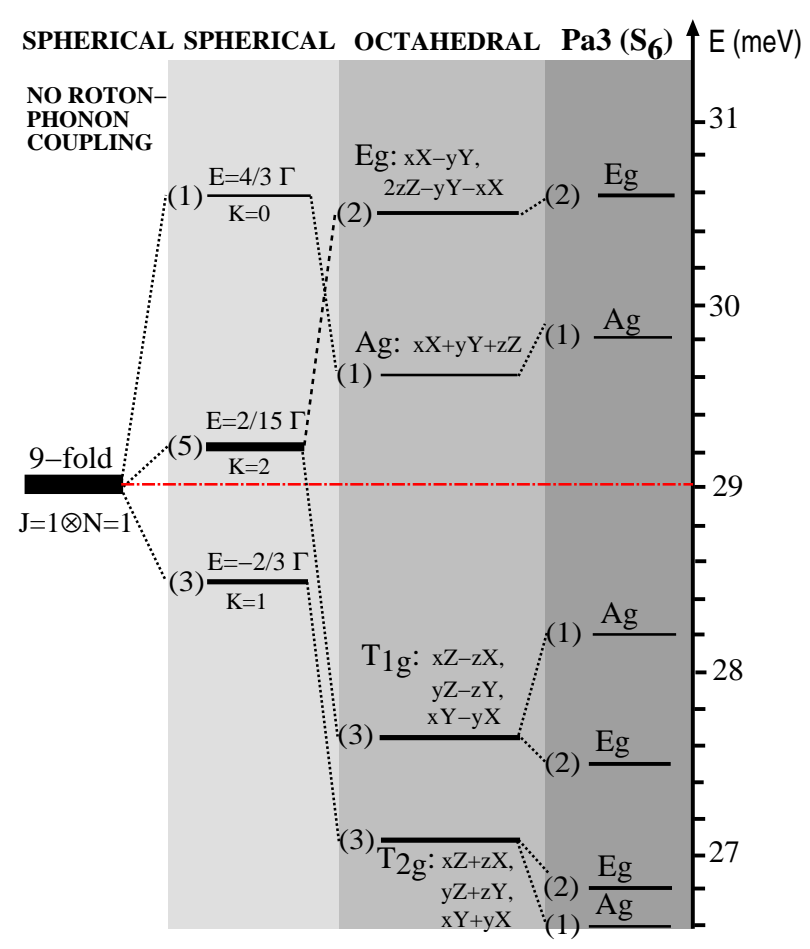

FIG. 2. Removal of degeneracy as roton-phonon interactions are introduced and the site symmetry is lowered. The degeneracy is indicated by the number in parenthesis. At the far left is shown the completely degenerate level when spherical symmetry is assumed and no roton-phonon coupling is present. The next panel shows the effect of allowing roton-phonon interactions but preserving overall spherical symmetry. Here $\mathrm{K}$ is the total (orbital plus orientational) angular momentum. In the next panel spherical symmetry is lowered to octahedral symmetry which is appropriate for $\mathrm{H}_{2}$ in the octahedral interstitial site in orientationally disordered $\mathrm{C}_{60}$. The far right panel (and the energy scale) applies to the case of $\mathrm{H}_{2}$ in orientationally ordered $\mathrm{C}_{60}$ in which case the site symmetry is $\mathrm{S}_{6}$. 


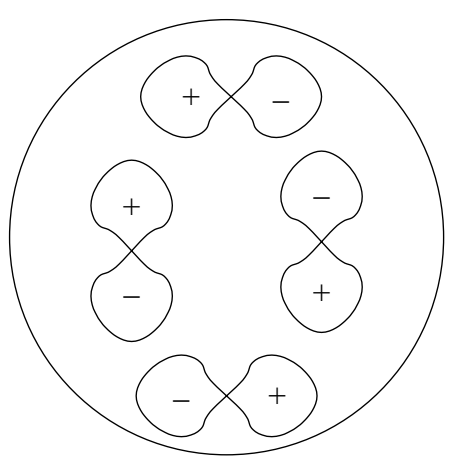

$$
\begin{aligned}
& \mid \mathrm{x} Y+\mathrm{y} X> \\
& \mathrm{K}=2, \mathrm{~T} 2 \mathrm{~g}
\end{aligned}
$$
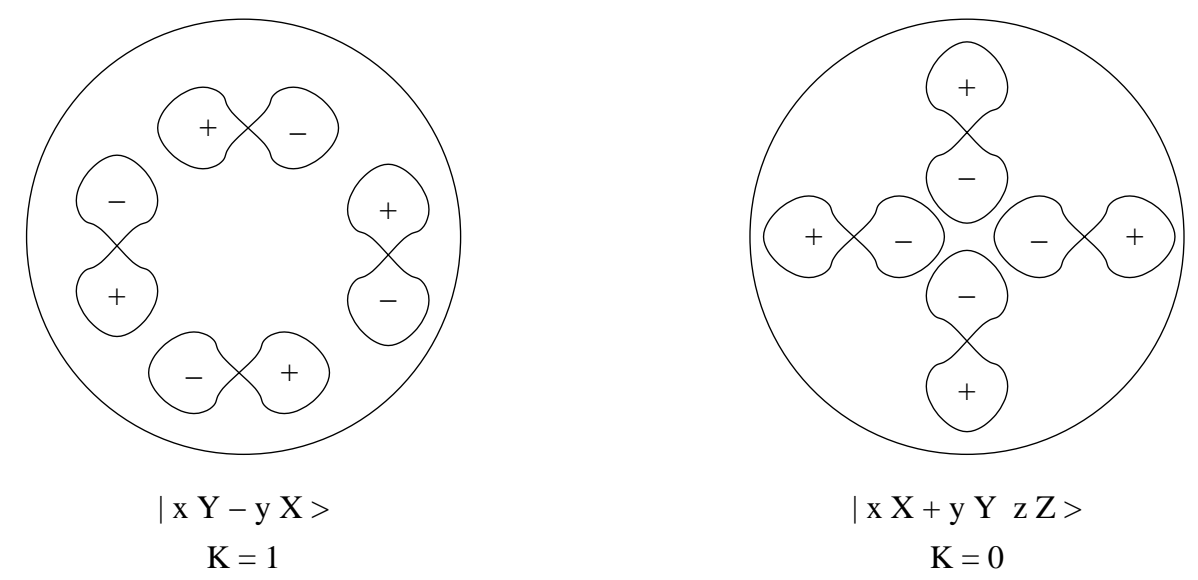

FIG. 3. Translation-rotation wavefunctions for a $(J=1) \mathrm{H}_{2}$ molecule in an octahedral interstitial site with one phonon. Here the plane of the paper is the $x-y$ plane and for simplicity only the dependence in this plane is depicted. Each figure eight represents an $|X\rangle$ or $|Y\rangle$ orientational wavefunction and the sign associated with each lobe of this $p$-like function is indicated. Each orientational wave function is multiplied by a translational wavefunction $|x\rangle,|y\rangle$, or $|z\rangle$, where for instance $|x\rangle \sim x \exp \left[-\frac{1}{4}(x / \sigma)^{2}\right]$. The presence of a phonon in the $r_{\alpha}$ coordinate thus causes the wave function to be an odd function of $r_{\alpha}$, as one sees in the diagrams. As indicated in Fig. 2, the total angular momentum, $K$, which is the sum of the angular momentum of the phonon and that of rotation, is a good quantum number whose value is indicated. Top, Left: a $K=2, \mathrm{~T}_{2 \mathrm{~g}}$ function, top right: a $K=2, E_{\mathrm{g}}$ function, bottom left: a $K=1, T_{1 \mathrm{~g}}$ function, and bottom right a $K=0, \mathrm{~A}_{\mathrm{g}}$ function. 


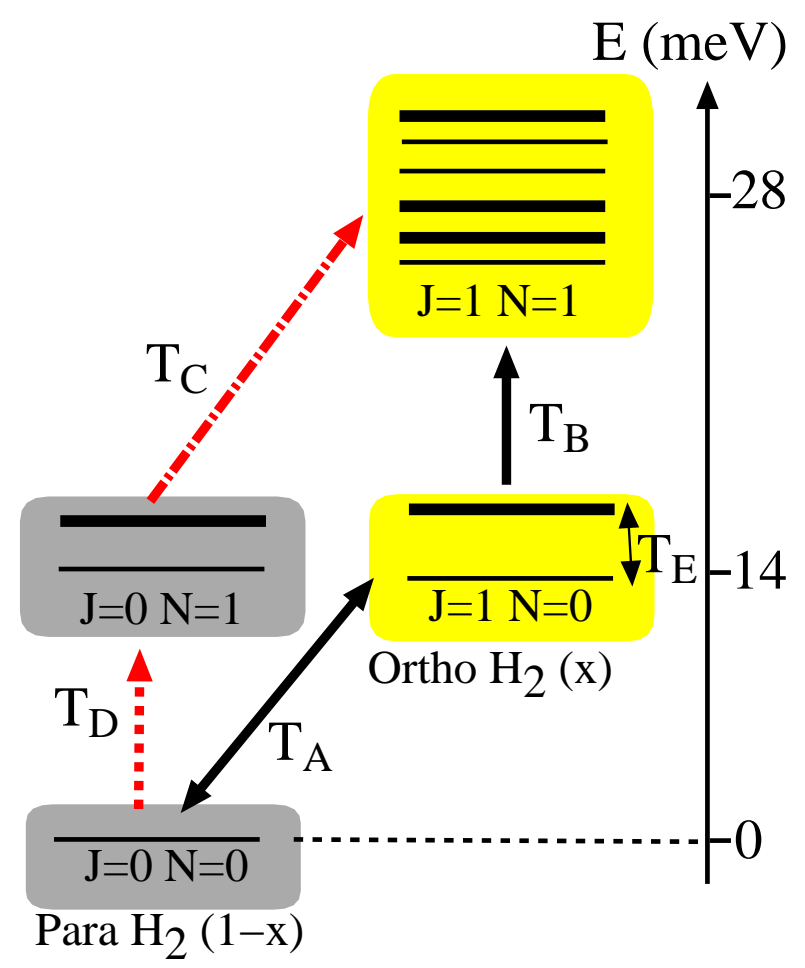

FIG. 4. A schematic representation of possible transitions between the rotation-phonon energy levels that could be observed in a neutron scattering experiment. At low temperature, only the $(J=0, N=0)$ and $(J=1, N=0)$ states are populated and therefore the transition $\mathrm{T}_{C}$ can not be observed at low temperature. The transition $\mathrm{T}_{D}$ is proportional to the coherent cross section of $\mathrm{H}_{2}$ and therefore very small. The transitions $\mathrm{T}_{B}$ and $\mathrm{T}_{A}$ have comparable cross sections (see text for details). 


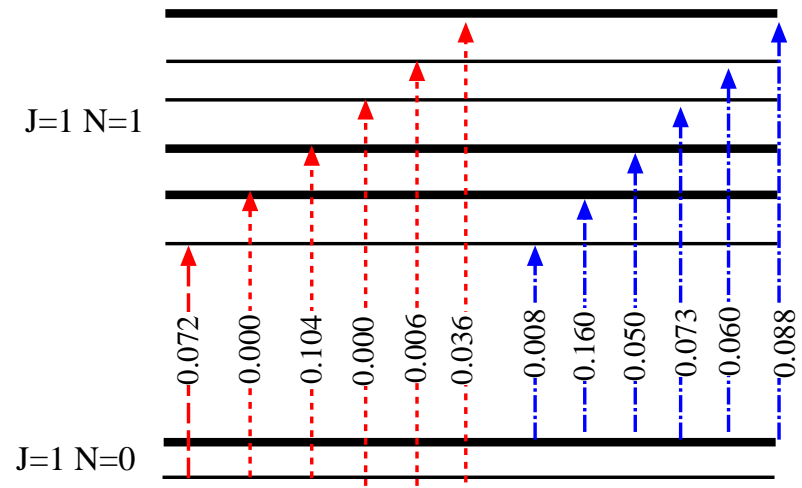

FIG. 5. The calculated transition probabilities from the $(J=1, N=0)$ levels to the nine-fold manifold of $(J=1, N=1)$ levels at $\mathrm{T}=4 \mathrm{~K}$. For each pair of energies these transition probabilities represent the appropriate sum over degenerate levels. Note that there are at least eight transitions with comparable probability, suggesting that rich features could be observed in a neutron scattering experiment. 


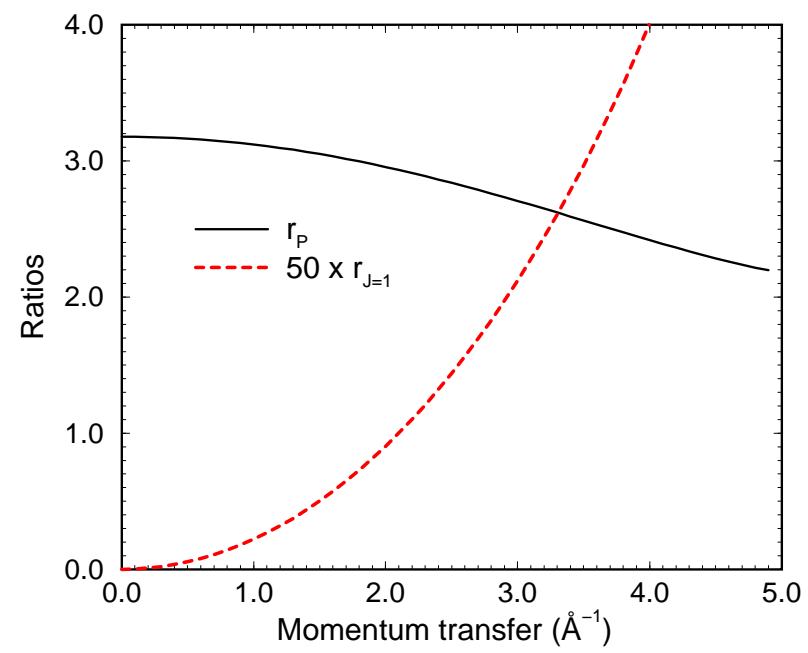

FIG. 6. The solid curve is the ratio $r_{P}$ of the single-phonon cross section to that from para-ortho conversion as given in Eq. (25) as a function of momentum transfer. The dotted line shows $50 \times r_{J=1}$ $(\mathrm{T}=0)$ as given in Eq. (26). The experimental situation of Ref. 5 corresponds to a momentum transfer between 2 and $4 \AA^{-1}$. 


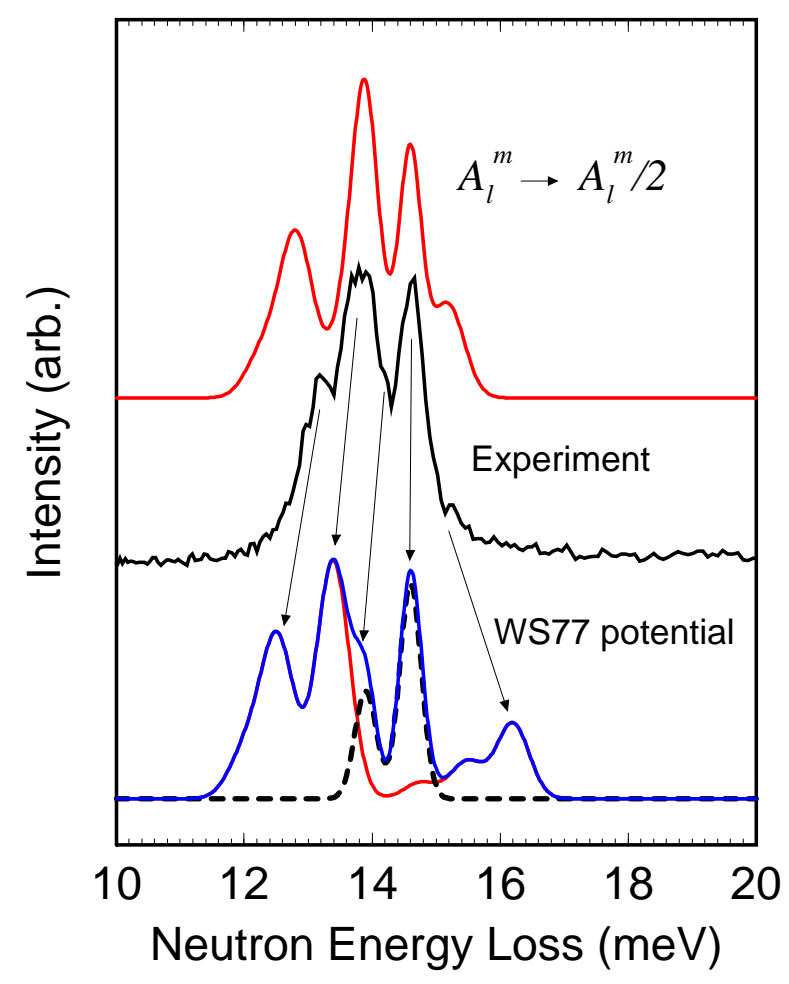

FIG. 7. Neutron energy loss spectrum (middle) at 4.2 K. The bottom curve is the result from our calculation using the WS77 potential. The dashed and gray lines are the contributions from rotational and vibrational excitations, respectively. The top curve is the spectrum after arbitrarily scaling $A_{l}^{m}$ by half, indicating that the orientational potential used in our calculations is too anisotropic as far as the center-of-mass motion is concerned. 


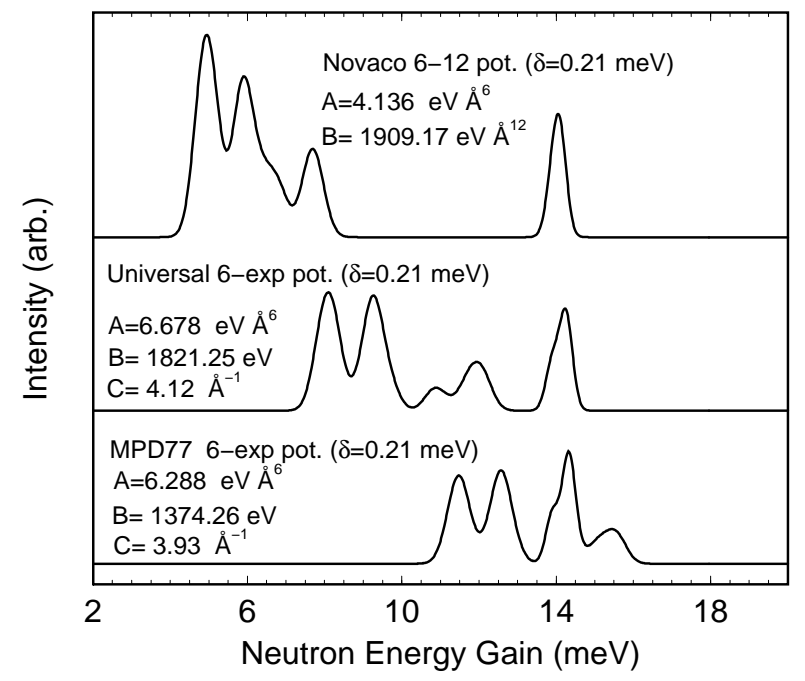

FIG. 8. Neutron energy loss spectrums obtained from various commonly used intermolecular potentials. For each potential we give the value of $\delta$, the splitting of the $(J=1)$ zero-phonon levels, which may be compared to the experimentally determined value $\delta=0.75 \mathrm{meV} .^{5}$ Note that the average phonon energies of the first two potentials (top and middle curves) are way off from the observed value of $\approx 14 \mathrm{meV}$. 


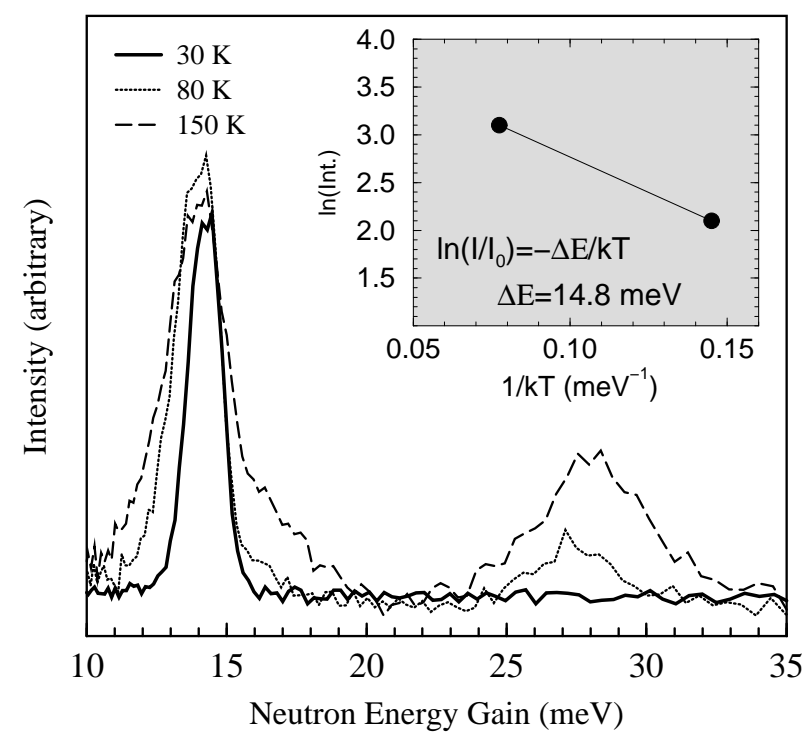

FIG. 9. Temperature dependent neutron energy gain spectrum of $\mathrm{H}_{2}$ in $\mathrm{C}_{60}$ (the data is taken from Ref. 5). The inset shows $\ln \left(I / I_{0}\right)$ versus $1 / k T$, where $I$ is the intensity of the feature at about $28 \mathrm{meV}$. The slope of the line indicates an activation energy barrier of $14.8 \mathrm{meV}$. 